\title{
Numerical Modeling Studies of The Dissolution-Diffusion-Convection Process During CO2 Storage in Saline Aquifers
}

\author{
Karsten Pruess and Keni Zhang \\ Earth Sciences Division, Lawrence Berkeley National Laboratory \\ University of California, Berkeley, CA 94720, U.S.A.
}

\begin{abstract}
For purposes of geologic storage, $\mathrm{CO} 2$ would be injected into saline formations at supercritical temperature and pressure conditions, and would form a separate phase that is immiscible with the aqueous phase (brine). At typical subsurface temperature and pressure conditions, supercritical $\mathrm{CO} 2$ ( $\mathrm{scCO} 2$ ) has lower density than the aqueous phase and would experience an upward buoyancy force. Accordingly, the $\mathrm{CO} 2$ is expected to accumulate beneath the caprock at the top of the permeable interval, and could escape from the storage formation wherever (sub-)vertical pathways are available, such as fractures or faults through the caprock, or improperly abandoned wells. Over time, an increasing fraction of $\mathrm{CO} 2$ may dissolve in the aqueous phase, and eventually some of the aqueous $\mathrm{CO} 2$ may react with rock minerals to form poorly soluble carbonates. Dissolution into the aqueous phase and eventual sequestration as carbonates are highly desirable processes as they would increase permanence and security of storage. Dissolution of $\mathrm{CO} 2$ will establish phase equilibrium locally between the overlying $\mathrm{CO} 2$ plume and the aqueous phase beneath. If the aqueous phase were immobile, $\mathrm{CO} 2$ dissolution would be limited by the rate at which molecular diffusion can remove dissolved $\mathrm{CO} 2$ from the interface between CO2-rich and aqueous phases. This is a slow process. However, dissolution of $\mathrm{CO} 2$ is accompanied by a small increase in the density of the aqueous phase, creating a negative buoyancy force that can give rise to downward convection of $\mathrm{CO} 2$-rich brine, which in turn can greatly accelerate $\mathrm{CO} 2$ dissolution. This study explores the process of dissolution-diffusionconvection (DDC), using high-resolution numerical simulation. We find that geometric features of convection patterns are very sensitive to small changes in problem specifications, reflecting self-enhancing feedbacks and the chaotic nature of the process. Total $\mathrm{CO} 2$ dissolution rates on the other hand are found to be quite robust against modest changes in problem parameters, and are essentially constant as long as no dissolved $\mathrm{CO} 2$ reaches the lower boundary of the system.
\end{abstract}




\section{Introduction}

$\mathrm{CO} 2$ injected into a saline aquifer can be stored in four different modes, (1) as a (mobile) separate supercritical phase, for simplicity here referred to as "free gas," (2) as trapped gas, (3) dissolved in the aqueous phase, and (4) as solid minerals (IPCC, 2005). At early times during a $\mathrm{CO} 2$ storage project, most of the injected $\mathrm{CO} 2$ will be present as free gas, but over time there is a continuous progression from storage mode (1) to (2), (3) and (4) (IPCC, 2005; Audigane et al., 2007). The quantitative aspects of this progression - the amounts of CO2 and the time scales involved - are of great interest, because storage security and permanence of storage increase as $\mathrm{CO} 2$ is first trapped, then dissolves, and eventually chemically binds to solid phases.

At typical subsurface conditions of interest, $\mathrm{CO} 2$ is less dense than the aqueous phase, and will therefore tend to rise to the top of the permeable formation into which it is injected. This "gravity override" may cause much of the available pore space to be bypassed, reducing storage capacity relative to what would be obtained in a uniform sweep (Nordbotten and Celia, 2006). $\mathrm{CO} 2$ accumulating beneath a caprock can access potential pathways for upward leakage, such as fracture zones and faults. $\mathrm{CO} 2$ will dissolve into the underlying aqueous phase, and will then be transported by molecular diffusion away from the interface between the two-phase (gas-aqueous) zone above into the single-phase aqueous zone below. Dissolution of $\mathrm{CO} 2$ will increase the density of the aqueous phase by a small amount that, depending on salinity, may range from 0.1 $1 \%$ (Fig. 1). The density increase is gravitationally unstable, and can give rise to downward convection of $\mathrm{CO} 2$-ladden water from the phase boundary. Such buoyant convection may greatly accelerate the rate at which $\mathrm{CO} 2$ is dissolved and removed from the overlying gas.

Because of its practical importance for $\mathrm{CO} 2$ storage, the process of dissolution-diffusionconvection (DDC) has been studied by many investigators. Here we make reference to selected studies that are of particular relevance to the present work. The earliest work that pointed out the significance of aqueous-phase density increase due to $\mathrm{CO} 2$ dissolution in the context of $\mathrm{CO} 2$ storage is by Weir and collaborators (Weir et al., 1995, 1996). Lindeberg and Bergmo (2003) addressed DDC and associated multi-scale resolution problems in the context of numerical simulations of the Sleipner Vest CO2 storage project in the Norwegian sector of the North Sea. 


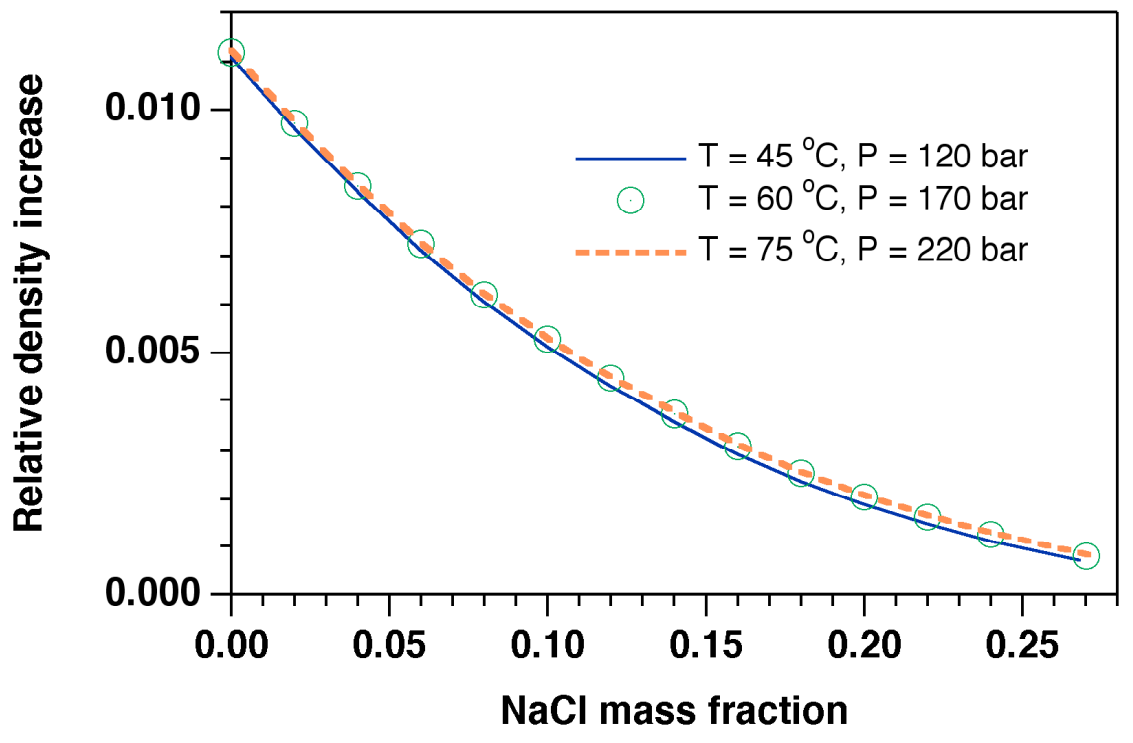

Figure 1. Relative density increase of sodium chloride brines due to dissolution of $\mathrm{CO} 2$, for typical temperature and pressure conditions encountered at storage depths from 1,000 - 2,000 m. The data were computed from correlations coded in the ECO2N fluid property module for the TOUGH2 simulator (Pruess and Spycher, 2007; Pruess, 2004).

Studies focusing on stability analyses for onset time for convection, preferred wavelength for growth of convective fingers, and growth rates were presented by Ennis-King et al. (2003a, b; 2005), Hesse et al. (2006), Riaz et al. (2006), Xu et al. (2006) and others. Convection induced by $\mathrm{CO} 2$ dissolution has similarities to thermally-buoyant convection, as driven by geothermal temperature gradients, and the extensive literature in that field (e.g. Garg and Kassoy, 1981) has helped understanding of convection induced by $\mathrm{CO} 2$ dissolution. There are important differences as well, the principal one being the prevalence of a constant (geothermal) temperature gradient as initial condition for thermal convection. This contrasts with the $\mathrm{CO} 2$ dissolution problem, in which there is no significant pre-existing gradient of dissolved aqueous $\mathrm{CO} 2$, making the system unconditionally unstable with respect to buoyant convection.

In the references cited above, the authors also present numerical simulations to complement and partially confirm analytical stability theory. Hesse et al. (2006) performed highresolution simulations for extended time periods, and found that total $\mathrm{CO} 2$ dissolution rate is 
constant during the convection-dominated period, apart from random fluctuations due to the chaotic nature of the underlying processes. Ennis-King et al. (2008) compared DDC for homogeneous media with simulations for heterogeneous media, that featured a random distribution of shale bodies in a homogeneous sand background. Recently, laboratory studies are being conducted that have confirmed qualitative and quantitative aspects of DDC (Yang and Gu, 2006; Kneafsey and Pruess, 2008).

This study explores quantitative aspects of $\mathrm{CO} 2$ dissolution. The DDC process begins on small spatial scales and, after convection is initiated, continually grows to eventually encompass the entire permeable interval. We employ high-resolution numerical simulation to examine the onset of convective activity, and the rate at which this process removes $\mathrm{CO} 2$ from the dissolution boundary. A first goal of our work is to achieve realistic numerical simulations of the DDC process, in which space and time are resolved on the actual scale of the governing diffusive and convective processes. A further goal, not addressed in this report, will be the design of sub-gridscale discretization methods that can achieve a satisfactory representation of the processes in a field-scale modeling approach. In the context of this goal, we are primarily interested in $\mathrm{CO} 2$ dissolution rates for systems whose vertical thickness $\mathrm{H}$ is large in comparison to the thickness $\mathrm{L}_{\mathrm{inc}}$ of the diffusive boundary layer at onset of instability, $\mathrm{H}>\mathrm{L}_{\mathrm{inc}}$. We focus on the earlier portion of the convective period, before convection is affected by the presence of a lower boundary. It is expected that late-stage convection, and systems with vertical thickness $\mathrm{H} \approx \mathrm{L}_{\text {inc }}$, can be treated adequately with the fairly coarse spatial resolutions that would be commonly employed in field-scale models.

\section{Molecular Diffusion}

Stability analysis has shown that, in the early stages, DDC is entirely dominated by molecular diffusion (Ennis-King and Paterson, 2003a, b). For a boundary concentration (CO2 mass fraction) $\mathrm{X}_{0}$, applied at time $\mathrm{t}=0$ to a homogeneous domain with initial concentration $\mathrm{X}=$ 0 , the concentration profile away from the boundary is given by the appropriate solution of the diffusion equation $\partial \mathrm{X} / \partial \mathrm{t}=\mathrm{D} \partial^{2} \mathrm{X} / \partial \mathrm{t}^{2}$ (Carslaw and Jaeger, 1959),

$$
\mathrm{X}(\mathrm{z}, \mathrm{t})=\mathrm{X}_{0} \operatorname{erfc}(\mathrm{u})
$$


where $u=z / 2 \sqrt{D t}$ is the similarity variable for the diffusion process, with $z$ the distance from the top boundary (measured positive downward), $t$ the time, and D the diffusivity. erfc(u) denotes the complementary error function, expressed in terms of the error function

$$
\operatorname{erf}(\mathrm{u})=\frac{2}{\sqrt{\pi}} \int_{0}^{u} \exp \left(-\xi^{2}\right) d \xi
$$

as $\operatorname{erfc}(\mathrm{u})=1-\operatorname{erf}(\mathrm{u})$. The thickness of the diffusive boundary layer can be estimated as $\Delta \mathrm{z}=\sqrt{\mathrm{Dt}}$, although it must be kept in mind that the diffusive profile is very broad, and significant non-zero concentrations extend beyond $\sqrt{\mathrm{Dt}}$ (see below). In some cases $2 \sqrt{\mathrm{Dt}}$ may be a better measure of the diffusive boundary layer, but for simplicity we will generally use $\sqrt{\mathrm{Dt}}$. A finite "incubation time" $t_{\text {inc }}$ must elapse before convection is initiated, estimated from linear stability analysis by Ennis-King and Paterson (2003a, b) as

$$
\mathrm{t}_{\text {inc }}=\mathrm{c}_{0} \frac{\mu^{2} \phi^{2} \mathrm{D}}{(\Delta \rho)^{2} \mathrm{~g}^{2} \mathrm{k}^{2}}
$$

Here, $\mu$ is the fluid viscosity, $\Delta \rho$ the density increase due to CO2 dissolution, $\phi$ is porosity, $\mathrm{k}$ permeability, and $\mathrm{g}$ acceleration of gravity. $\mathrm{c}_{0}$ is a numerical constant that was estimated as $\mathrm{c}_{0} \approx$ 180 based on the critical Rayleigh number for onset of convective instability, while from numerical simulations a value of $\mathrm{c}_{0}=80-100$ was obtained (Ennis-King and Paterson, 2003b). Our simulations yield much larger values of $\mathrm{c}_{0}>1,000$, see below. The thickness of the diffusive boundary layer at the onset time $t_{\text {inc }}$ of convective activity is given by

$$
\mathrm{L}_{\mathrm{inc}}=\sqrt{\mathrm{Dt}_{\mathrm{inc}}}=\sqrt{\mathrm{c}_{0}} \frac{\mu \phi \mathrm{D}}{(\Delta \rho) \mathrm{gk}}
$$

From the expression for diffusive flux, 


$$
\mathrm{F}(\mathrm{z}, \mathrm{t})=-\phi \rho \mathrm{D} \frac{\partial \mathrm{X}}{\partial \mathrm{z}}=\phi \rho \mathrm{X}_{0} \sqrt{\frac{\mathrm{D}}{\pi \mathrm{t}}} \exp \left(-\frac{\mathrm{z}^{2}}{4 \mathrm{Dt}}\right)
$$

the rate of $\mathrm{CO} 2$ uptake at the dissolution boundary $(\mathrm{z}=0)$, per unit cross-sectional area, can be written as

$$
\mathrm{F}(\mathrm{z}=0, \mathrm{t})=\mathrm{F}(\mathrm{t})=\phi \rho \mathrm{X}_{0} \sqrt{\mathrm{D} / \pi \mathrm{t}}
$$

Substituting Eq. (3) into Eq. (6), we obtain the diffusive flux at the onset time of convection as

$$
\mathrm{F}\left(\mathrm{z}=0, \mathrm{t}_{\mathrm{inc}}\right)=\frac{\mathrm{X}_{0} \rho \Delta \rho \mathrm{gk}}{\mu \sqrt{\pi \mathrm{c}_{0}}}
$$

indicating that this flux is independent of porosity and diffusivity. The total dissolved $\mathrm{CO} 2$ inventory accumulated after time t per unit cross-sectional area can be obtained by integrating Eq. (6) to yield

$$
M(t)=2 \phi \rho X_{0} \sqrt{\frac{D t}{\pi}}
$$

Eqs. $(6,8)$ imply that, for a purely diffusive process, the quantity F.M will be a constant, independent of time

$$
\mathrm{F} \cdot \mathrm{M}=\mathrm{F}(\mathrm{t}) \cdot \mathrm{M}(\mathrm{t})=\dot{\mathrm{M} M}=\frac{1}{2} \frac{\mathrm{d}}{\mathrm{dt}}\left(\mathrm{M}^{2}\right)=\frac{2}{\pi} \phi^{2} \rho^{2} \mathrm{X}_{0}^{2} \mathrm{D}
$$

As will be discussed below, F.M grows approximately linearly with time for a convective process, providing a sensitive measure for the convective onset time $\mathrm{t}_{\mathrm{inc}}$. 
Of interest is the total solute inventory per unit cross-sectional area at time $t$ in the interval $(0, \mathrm{z})$. By integrating Eq. (1), the fraction of total solute inventory in the interval $0 \leq \mathrm{u} \leq \mathrm{z} / 2 \sqrt{\mathrm{Dt}}$ can be written as

$$
f=\frac{M(u)}{M(\infty)}=\sqrt{\pi} \int_{0}^{u} \operatorname{erfc}(x) d x
$$

where we have used $\int_{0}^{\infty} \operatorname{erfc}(\mathrm{x}) \mathrm{dx}=1 / \sqrt{\pi}$ (Carslaw and Jaeger, 1959). From the formulas and tabulations provided by Carslaw and Jaeger (1959), Table 1 gives the relative concentrations $\mathrm{C}=$ $\mathrm{X} / \mathrm{X}_{0}$ and fractional solute inventory for two commonly employed measures of diffusive penetration.

Table 1. Relative concentrations and fractional solute inventory in a diffusive profile.

\begin{tabular}{|c|c|c|c|}
\hline $\begin{array}{c}\text { similarity } \\
\text { variable } \\
\mathrm{u}\end{array}$ & $\begin{array}{c}\text { distance } \\
\mathrm{z}\end{array}$ & $\begin{array}{c}\text { relative } \\
\text { concentration } \\
\mathrm{C}\end{array}$ & $\begin{array}{c}\text { fractional } \\
\text { inventory } \\
\mathrm{f}\end{array}$ \\
\hline 0.5 & $\sqrt{\mathrm{Dt}}$ & 0.4795 & 0.6461 \\
\hline 1.0 & $2 \sqrt{\mathrm{Dt}}$ & 0.1573 & 0.9109 \\
\hline
\end{tabular}

\section{Numerical Simulations}

\subsection{Approach}

$\mathrm{CO} 2$ injected into a saline aquifer is buoyant, and will tend to migrate towards the top of the permeable formation, as schematically shown in Fig. 2. In the present study we do not address the full field-scale problem; instead, we focus on a vertical cross section beneath the two-phase zone. The upper boundary of the single-phase aqueous region remains at saturated $\mathrm{CO} 2$ concentrations, due to the overlying free supercritical $\mathrm{CO} 2$ phase. This is modeled by maintaining a small free gas saturation at the top boundary of the flow domain (see Fig. 3). 


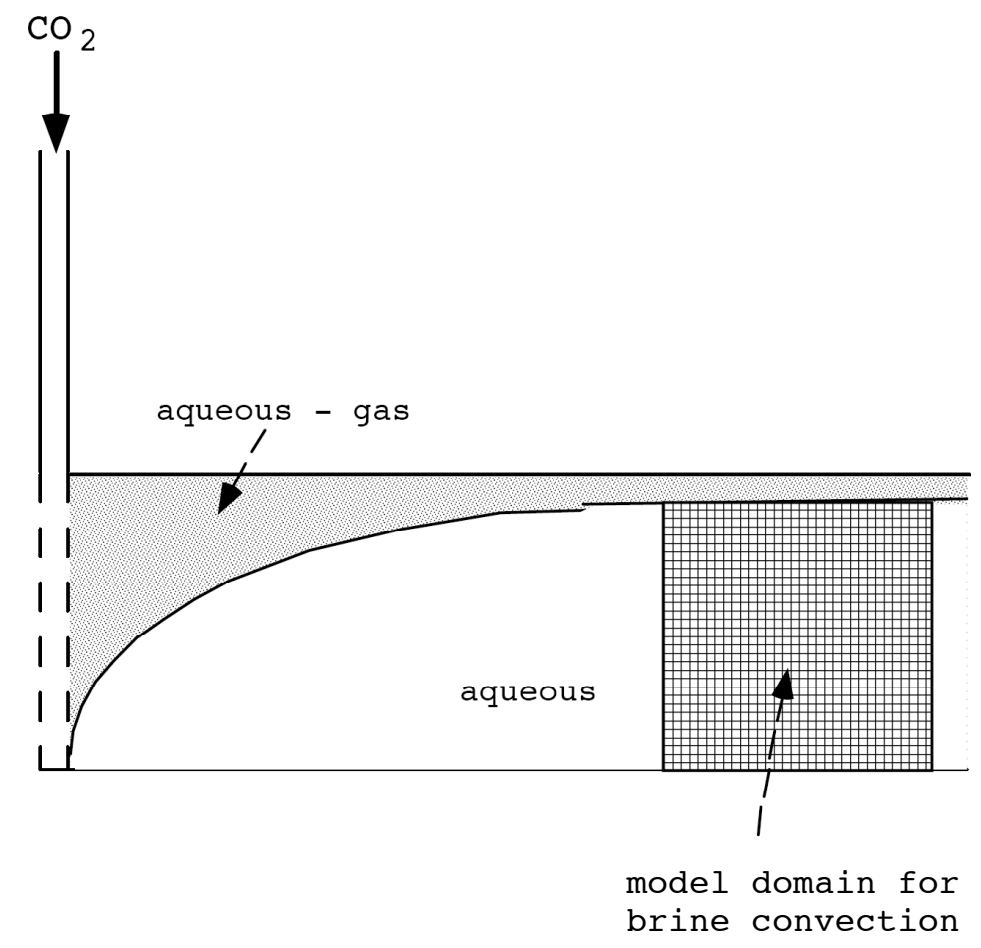

Figure 2. Schematic of phase distributions around a CO2 injection well.

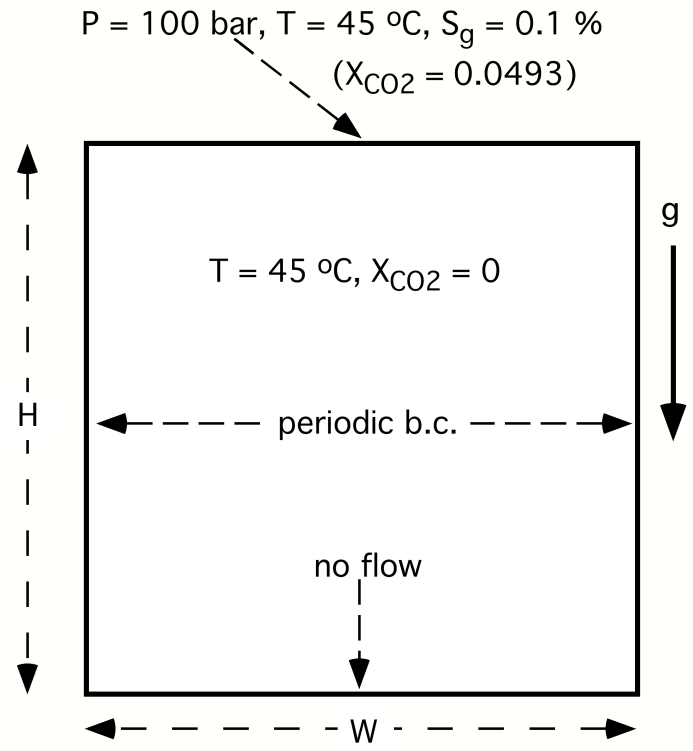

Figure 3. Schematic of specifications used for numerical simulations. Different heights $\mathrm{H}$ and widths $\mathrm{W}$ were employed for the simulation domain. Periodic boundary conditions on the left and right are explained in the text. 
Dissolved CO2 will be removed from the interface between the two-phase zone above and single-phase aqueous conditions below by molecular diffusion at early times, and additionally by convective transport at times $\mathrm{t}>\mathrm{t}_{\mathrm{inc}}$. Dissolved concentration in the interface is maintained constant due to contact with the overlying gas phase; therefore, the rate at which $\mathrm{CO} 2$ is transported into the single-phase aqueous region is equal to the rate at which $\mathrm{CO} 2$ dissolves at the phase boundary. The CO2 dissolution rate per unit "footprint area" of the reservoir is the most important quantity characterizing the DDC process, and in our simulations is computed as (space-averaged) $\mathrm{CO} 2$ flux across the top boundary of the model. We also employ other "integral" measures of the DDC process, including onset time of convection $\mathrm{t}_{\mathrm{inc}}$, and the growth of total dissolved $\mathrm{CO} 2$ inventory over time.

The flow system is initialized in gravity equilibrium (hydrostatic pressure gradient) relative to the top boundary. For the simulation of the $\mathrm{CO} 2$ dissolution-diffusion-convection process, we suppress advection of $\mathrm{CO} 2$-ladden water from the top boundary. The porous medium is assumed homogeneous, but a random variation of permeabilities with an amplitude of $\pm 1 \%$ is superimposed to seed the convective activity. The sensitivity of DDC to different random seeds is explored. Isothermal conditions are assumed throughout. In order to avoid unphysical effects from the limited size of the domain modeled, we apply "periodic" boundary conditions at the lateral edges. This is accomplished by connecting the grid blocks on the right hand side with those on the left hand side in Fig. 3, which is tantamount to "rolling up" the section about a vertical axis so that the right and left boundaries touch, in effect modeling flow on the mantle of a cylinder instead of in a rectangle. The bottom boundary is usually modeled as "no flow." The vertical extent of the domain limits the time for which the convective activity can be modeled before effects of the lower boundary will be felt. Some variations in boundary conditions, domain size, and space discretization were explored, in order to study the sensitivity of system behavior to these parameters.

We neglect chemical reactions, so that dissolved $\mathrm{CO} 2$ acts as a conservative solute tracer. Our initial numerical simulations were done with TOUGH2/ECO2N, which includes an accurate representation of thermophysical properties of mixtures of $\mathrm{NaCl}$ brine and $\mathrm{CO} 2$ (Pruess, 2004; Pruess and Spycher, 2007). TOUGH2/ECO2N represents the fluid as a 3-component mixture of 
$\mathrm{H} 2 \mathrm{O}, \mathrm{NaCl}$, and $\mathrm{CO} 2$. For conditions limited to a single aqueous phase, a more efficient calculation can be accomplished with TOUGH2/EOS7, in which the fluid is represented as a two-component mixture of $\mathrm{H} 2 \mathrm{O}$ and brine; the latter being used to represent density changes in the aqueous phase from $\mathrm{CO} 2$ dissolution. Numerical work is approximately proportional to $\mathrm{NEQ}^{2}$, where NEQ is the number of equations per grid block. Accordingly, a given problem can be solved with EOS7 $(\mathrm{NEQ}=2)$ in less than half the time required with ECO2N $(\mathrm{NEQ}=3)$. Test calculations have confirmed excellent agreement between simulations using EOS7 and ECO2N.

In order to achieve calculations with "sufficient" spatial resolution (see below) for domains of reasonable size, and over "reasonable" time scales, we use a parallelized version of TOUGH2 running on a dual-quad core PC (Wu et al., 2002; Zhang et al., 2008). In performing a parallel simulation, the TOUGH2-MP code first subdivides a simulation domain, defined by a TOUGH2 grid, into a number of subdomains by using the partitioning algorithm from the METIS software package (Karypsis and Kumar, 1998). The parallel code then relies on the MPI (Message-Passing Interface; Message Passing Forum, 1994) for its parallel implementation. Parallel simulations are run as multiple processes on several or many processors simultaneously. Each process involves calculations for one portion of the simulation domain to update thermophysical properties, assemble mass and energy balance equations, solve linear equation systems, and perform other local computations. The local linear equation systems are solved in parallel as multiple processes with the Aztec linear solver package (Tuminaro et al., 1999). When running TOUGH2-MP on multi-core PCs, or massively-parallel supercomputers, we normally would associate each processor (core or CPU) with one process. However, test calculations showed that in many cases super-linear speedup could be achieved, due to large efficiency gains for the linear equation solution when the flow domain is partitioned into a larger number of smaller subdomains (Zhang et al., 2007). This can make it advantageous to define a larger number of processes than are available as hardware processors. The simulations presented here were performed an a Dell T5400 dual quad core computer with a total of 8 cores, and in most cases 16 processes were run (two per processor), as this was found to reduce total execution time compared to running 8 processes. Depending on grid resolution and simulation time, individual runs typically took from $1 / 2$ to 4 hours. 


\subsection{Space Discretization}

Evolution and interaction of flow and transport over a broad range of space and time scales is an essential part of the problem studied here. We have not attempted any formal analysis of the space and time truncation errors associated with limitations in spatial and temporal resolution, because the convective behavior is subject to self-enhancing instabilities, is chaotic in nature (Kimura et al., 1986), and cannot be tackled with conventional concepts of "grid convergence" and "time truncation stability." We have followed a heuristic approach, performing numerical experiments with different grid resolution and time step controls, and comparing and contrasting observed system behavior with variability arising from variations in problem specifications (e.g., boundary conditions, and random seeding of permeability heterogeneity). Grid resolution is deemed adequate when gradients of dissolved $\mathrm{CO} 2$ concentrations are resolved, and "integral" measures of the $\mathrm{CO} 2$ transport process are stabilized. This can be judged from the outcome of our numerical simulation experiments. As will be seen below, "integral" measures of the DDC process, such as total CO2 dissolution rate at a phase boundary, tend to be rather robust, with respect to variations in both numerical discretization and overall parameter specifications of the flow system. This is an encouraging result, as it is the total $\mathrm{CO} 2$ dissolution rates that are of primary interest in connection with permanence and security of $\mathrm{CO} 2$ storage.

The early-time diffusive behavior can provide useful guidance for the spatial resolution required in space-discretized numerical models to be able to resolve the concentration gradients that drive the process. Depending primarily on formation permeability (see Eq. 3), incubation times for onset of convection in field systems may range from $10^{5}$ to $10^{9} \mathrm{~s}$ or more ( 1 day to 30 years; Ennis-King et al., 2005). For a nominal effective aqueous diffusivity of $10^{-9} \mathrm{~m}^{2} / \mathrm{s}$ of dissolved $\mathrm{CO} 2$, these times correspond to a diffusive boundary layer thickness $\mathrm{L}_{\text {inc }}$ in the range of $0.01-0.3 \mathrm{~m}$, necessitating $\mathrm{mm}$ - to $\mathrm{cm}$-level space discretization near the top (dissolution) boundary. Similar arguments can be made to estimate the thickness, and associated space discretization requirements, of the diffusive halo that will evolve as convective "fingers" or "tongues" proceed downward. 
Space resolution requirements can also be considered in the context of the likely practical needs for accuracy and time resolution of field-scale models of CO2 storage systems. During the active (injection) phase, which may last of the order of 30 years, "reasonable" accuracy will be demanded on a 1-year time scale, in turn requiring temporal resolution of order 1 month. For a diffusive process with diffusivity of order $10^{-9} \mathrm{~m}^{2} / \mathrm{s}$, the corresponding penetration depth is $5 \mathrm{~cm}$. Convective processes can cover comparable or larger distance. From Darcy's law, volumetric flux associated with buoyancy flow for typical parameters applicable to CO2 storage (see Table 2) can be estimated as $\mathrm{u}=(\mathrm{k} / \mu)(\Delta \rho \mathrm{g} / 2) \approx 10^{5} \mathrm{k}$, corresponding to pore velocities $\mathrm{v}=\mathrm{u} / \phi$ in the range of $1-100 \mathrm{~m} / \mathrm{yr}$ for permeabilities of $10^{-13}-10^{-11} \mathrm{~m}^{2}$. Propagation distances per month will then be $0.1-10 \mathrm{~m}$.

The convective activity initiated after the onset time $t_{\text {inc }}$ increases the rate at which solute is transported away from the boundary. Such an increase is possible only if the diffusive boundary layer becomes thinner on average after time $t_{\text {inc }}$ has elapsed. In fact, while downward convection of CO2-ladden water will transport water with larger dissolved concentrations to greater depth, a concomitant upward convection of water low in $\mathrm{CO} 2$ will be induced, that will reduce the thickness of the diffusive boundary layer locally, and will promote increased rates of diffusive removal of solute from the upper boundary. These considerations suggest a critical role of convectively-induced concentration changes for mass transport in the diffusive boundary layer, suggesting that space discretization requirements there are likely to be more severe than estimated for a diffusion-only process. From a practical viewpoint, if grid sizes are to be limited to $10^{5}$ grid blocks or less and relevant gradients are to be resolved properly, analysis of a highpermeability system $\left(\mathrm{k}=10^{-11} \mathrm{~m}^{2}\right)$ in 2-D is limited to a domain size of order $1 \mathrm{~m}$, while for a system with a lower permeability of $\mathrm{k}=10^{-13} \mathrm{~m}^{2}$, an order of magnitude larger scale can be modeled.

Away from the top boundary, it may not be necessary to fully resolve convective processes in order to get a reasonable approximation to field-scale behavior. Indeed, the overall rate of convective flow in a given region depends mainly on the total solute inventory in that region, and only weakly on the manner in which the solute is distributed. Accordingly, it may be possible to model overall convection rates with reasonable accuracy, even if individual 
convective tongues are poorly resolved. Space resolution requirements are more stringent for solute transport, which is governed by a hyperbolic PDE, than for overall aqueous phase flow, which is governed by a PDE that is parabolic or even elliptic (if aqueous phase is assumed incompressible). Errors arising in transport simulations with coarse gridding in the main flow direction (vertical) will be subject to some self-compensation over extended time periods, with solute breakthrough curves (BTCs) at a given horizon more dispersed for coarse grids, but approaching the same cumulative solute flow at later times. The dispersion length is given by the applied grid spacing h.

We now present numerical simulations for two sets of parameters, a "high-permeability" Case $1\left(\mathrm{k}=10^{-11} \mathrm{~m}^{2} \approx 10 \mathrm{D}\right)$, and a "moderate permeability" Case $2\left(\mathrm{k}=10^{-13} \mathrm{~m}^{2} \approx 100 \mathrm{mD}\right)$; in both cases, permeability is assumed isotropic. In presenting results we will emphasize total CO2 flux at the top boundary of the flow domain; as had been noted above, this quantity is numerically equal to the rate at which $\mathrm{CO} 2$ is dissolved. Here and in the following we always refer $\mathrm{CO} 2$ dissolution rates and dissolved inventories to $1 \mathrm{~m}^{2}$ of footprint area. This makes results for different-size model domains directly comparable, and allows quantities to be easily scaled for $\mathrm{CO} 2$ plume areas of interest.

\section{Results for Case 1 (high-k, small scale)}

This case uses a large formation permeability of $10^{-11} \mathrm{~m}^{2}$ (10 Darcy), as may be appropriate for well-sorted sands, and also for typical laboratory sandpacks. Problem specifications are given in Table 2. Due to the large permeability, time scales and thickness of the diffusive boundary layer are shifted to small values, see Eqs. (3, 4). Fig. 4a shows results for $\mathrm{CO} 2$ flux from the top boundary, which is equal to total $\mathrm{CO} 2$ dissolution rate per unit area. This and subsequent simulations used a random number seed of 0.8. At early times, $\mathrm{CO} 2$ inventory shows the characteristic $\sqrt{\mathrm{t}}$ dependence for diffusion (Fig. 4b). The data shown in Fig. 4 were obtained with two different levels of vertical discretization, using either a uniform grid resolution of $\mathrm{h}=10 \mathrm{~mm}$, or a grid that near the top boundary is refined to $\mathrm{h}=1 \mathrm{~mm}$. Horizontal grid resolution is $10 \mathrm{~mm}$. It is seen that the coarser grid initially underestimates and later overestimates diffusive flux. Agreement with the analytically computed diffusive flux is good from about $1.1-1.4 \times 10^{5} \mathrm{~s}$, and subsequently simulated fluxes become larger, reflecting increased 
Table 2. Parameters used for Case 1 simulations.

\begin{tabular}{|l|l|}
\hline Fluid properties & \\
\hline temperature & $\mathrm{T}=45^{\circ} \mathrm{C}$ \\
pressure & $\mathrm{P}=100 \mathrm{bar}$ \\
salinity & $\mathrm{X}_{\mathrm{s}}=0$ \\
dissolved $\mathrm{CO} 2$ concentration & $\mathrm{X}=0$ \\
$\begin{array}{l}\text { viscosity } \\
\text { water density } \\
\text { dissolved } \mathrm{CO}_{2} \text { mass fraction } \\
\text { at the top boundary }\end{array}$ & $\mu=0.5947 \times 10^{-3} \mathrm{~Pa}-\mathrm{s}$ \\
$\begin{array}{l}\text { density increase of aq. phase } \\
\text { from } \mathrm{CO}_{2} \text { dissolution }\end{array}$ & $\mathrm{X}_{0}=0.049306$ \\
$\begin{array}{l}\text { diffusivity } \\
\text { Formation properties }\end{array}$ & $\Delta \rho=10.45 \mathrm{~kg} / \mathrm{m}^{3}$ \\
\hline $\begin{array}{l}\text { porosity } \\
\text { permeability }\end{array}$ & $\mathrm{D}=2 \times 10^{-9} \mathrm{~m}^{2} / \mathrm{s}$ \\
\hline Model domain & $\mathrm{k}=0.3$ \\
\hline $\begin{array}{l}\text { height } \\
\text { width }\end{array}$ & $\mathrm{k}=10^{-11} \mathrm{~m}^{2}$ \\
\hline
\end{tabular}

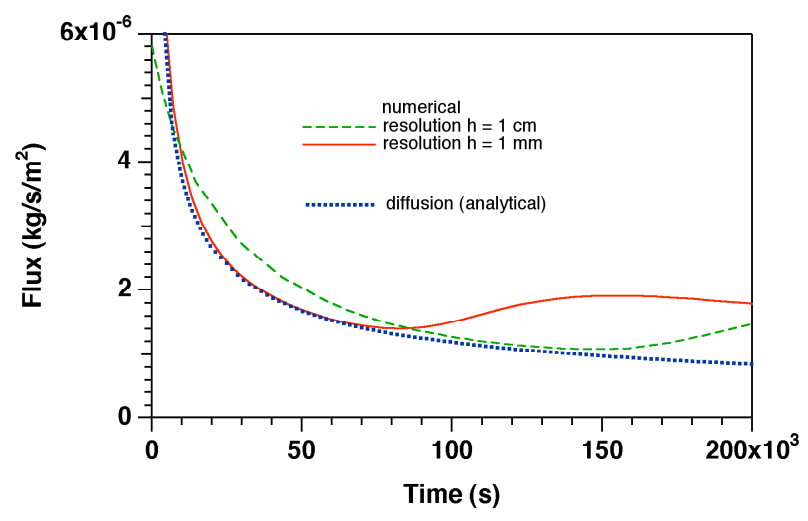

(a)

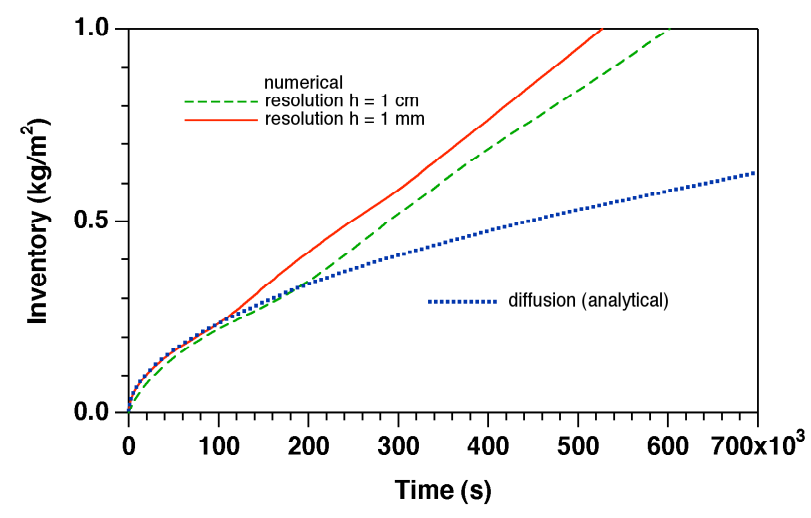

(b)

Figure 4. $\mathrm{CO} 2$ flux from the top boundary (a) and $\mathrm{CO} 2$ inventory (b).

$\mathrm{CO} 2$ uptake due to convective activity. The simulation with a finer vertical grid resolution of $\mathrm{h}=$ $1 \mathrm{~mm}$ near the top boundary agrees very well with the analytical diffusive flux until about $7 \times 10^{4}$ s, indicating an earlier onset of convective activity than seen with the coarser grid. Dissolved $\mathrm{CO} 2$ inventory, being an integral quantity, does not significantly exceed the inventory for the diffusion-only case until times well in excess of $t_{\text {inc }}=7 \times 10^{4} \mathrm{~s}$, thus providing a less sensitive measure for $\mathrm{t}_{\text {inc }}$ than $\mathrm{CO} 2$ fluxes (Fig. $4 b$ ). 
The good match for the diffusive behavior indicates that a grid resolution of $h=1 \mathrm{~mm}$ near the top boundary is adequate for the vertical direction. Horizontal resolution is an issue as well, as this places a lower limit on the wavelength of convective fingers that can be resolved. Theoretical analysis has shown that finger wavelengths favored for growth during the initial period of the convective instability are comparable to the thickness of the diffusive boundary layer at onset of convection (Ennis-King et al., 2005). For a convective onset time of $t_{\text {inc }}=7 \times 10^{4}$ $\mathrm{s}$, the boundary layer thickness is $|\mathrm{z}|=\mathrm{L}_{\mathrm{inc}}=\sqrt{\mathrm{Dt}_{\mathrm{inc}}}=11.8 \mathrm{~mm}$. Accordingly, we chose a horizontal grid resolution of $10 \mathrm{~mm}$. Corroboration of this choice comes from the numerical simulation results. Fig. 5 shows dissolved $\mathrm{CO} 2$ concentrations at different times, illustrating the onset and evolution of convective activity. The first frame corresponds to a time only slightly larger than the onset time of convection. The diffusive boundary layer has been distorted by numerous convective fingers; at the earliest time shown in Fig. 5 there are 25 fingers, indicating a wavelength of $40 \mathrm{~mm}$, a factor 4 larger than grid spacing. Subsequently the convective fingers advance downward, and in so doing become broader and partially coalesce and merge. This process continues as time progresses (see also frames at later time in Figs. 12a and 13a), leaving only two broad fingers at the time when significant $\mathrm{CO} 2$ concentrations approach the bottom boundary (Fig. 13a). These simulation results suggest that a horizontal grid spacing of $10 \mathrm{~mm}$ is adequate for resolving the horizontal scale of the fingers.

As a further test of grid resolution, additional runs were made using the SCALE facility in TOUGH2 to change all linear dimensions of the computational grid by factors ranging from 0.2 - 3.0. This linear scaling offers a very simple and convenient way to examine grid resolution effects by means of a single input parameter, and avoids the tedium of generating new grids with different resolution. A limitation is that the SCALE factor applies equally to horizontal and vertical grid dimensions, so that effects of vertical and horizontal grid resolution cannot be separated. (Another limitation is that the flow domain itself shrinks or expands according to the SCALE factor. However, here we focus on the early-time behavior during the initial onset of convection, for which effects of distant boundaries are negligible, see below.) Fig. 6 shows CO2 dissolution rates for SCALE factors in the range from 0.2 - 3.0. It is seen that over this entire parameter range, the dissolution rates agree well with the analytically calculated diffusive flux at 
early times. We conclude that vertical grid resolution in the range from $0.2-3.0 \mathrm{~mm}$ is adequate for resolving the early-time diffusive process.
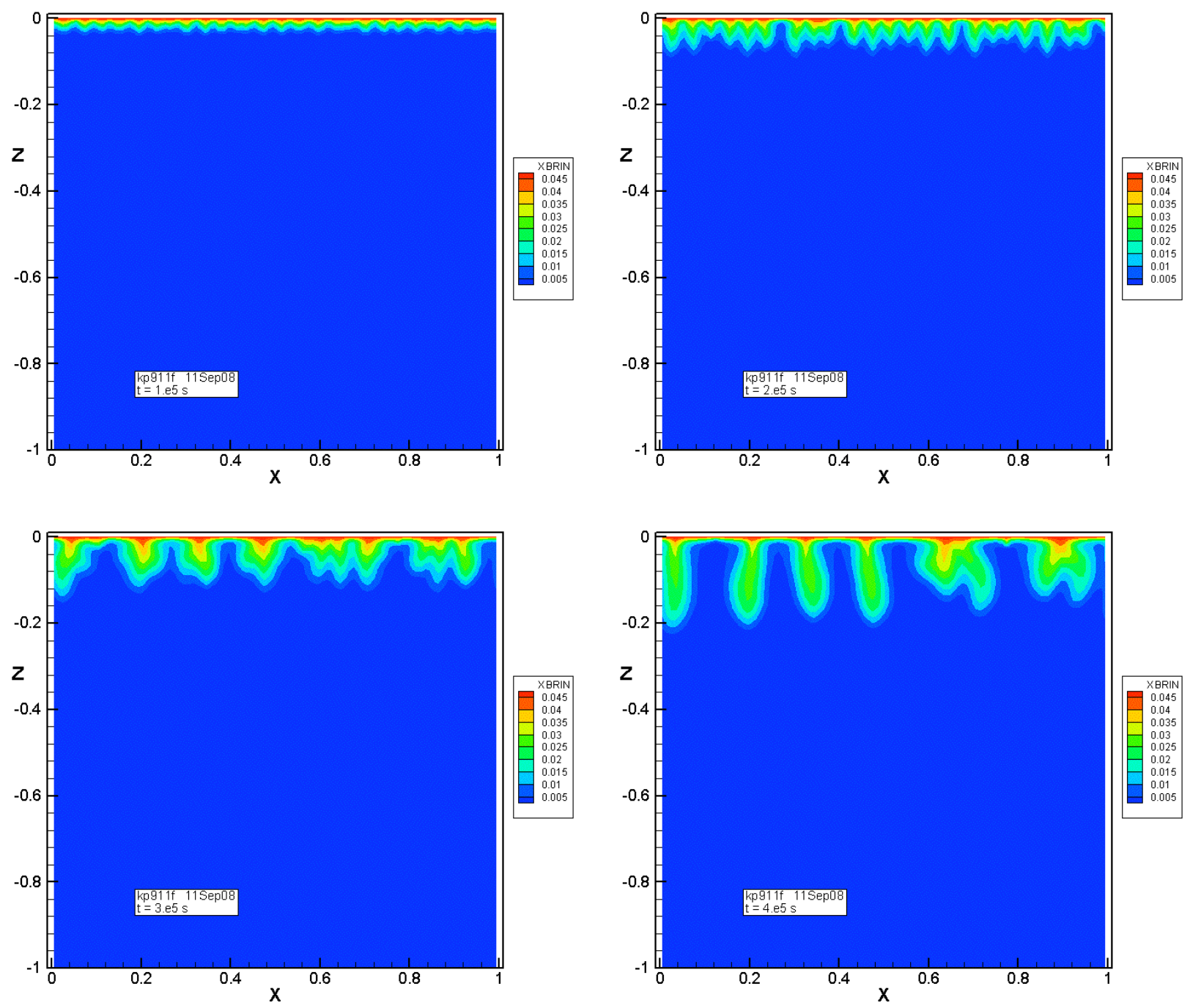

Figure 5. Dissolved CO2 concentrations, expressed as mass fractions XBRIN, at different times for Case 1, obtained with the fine grid. Here and in subsequent frames, dimensions are in meters. 


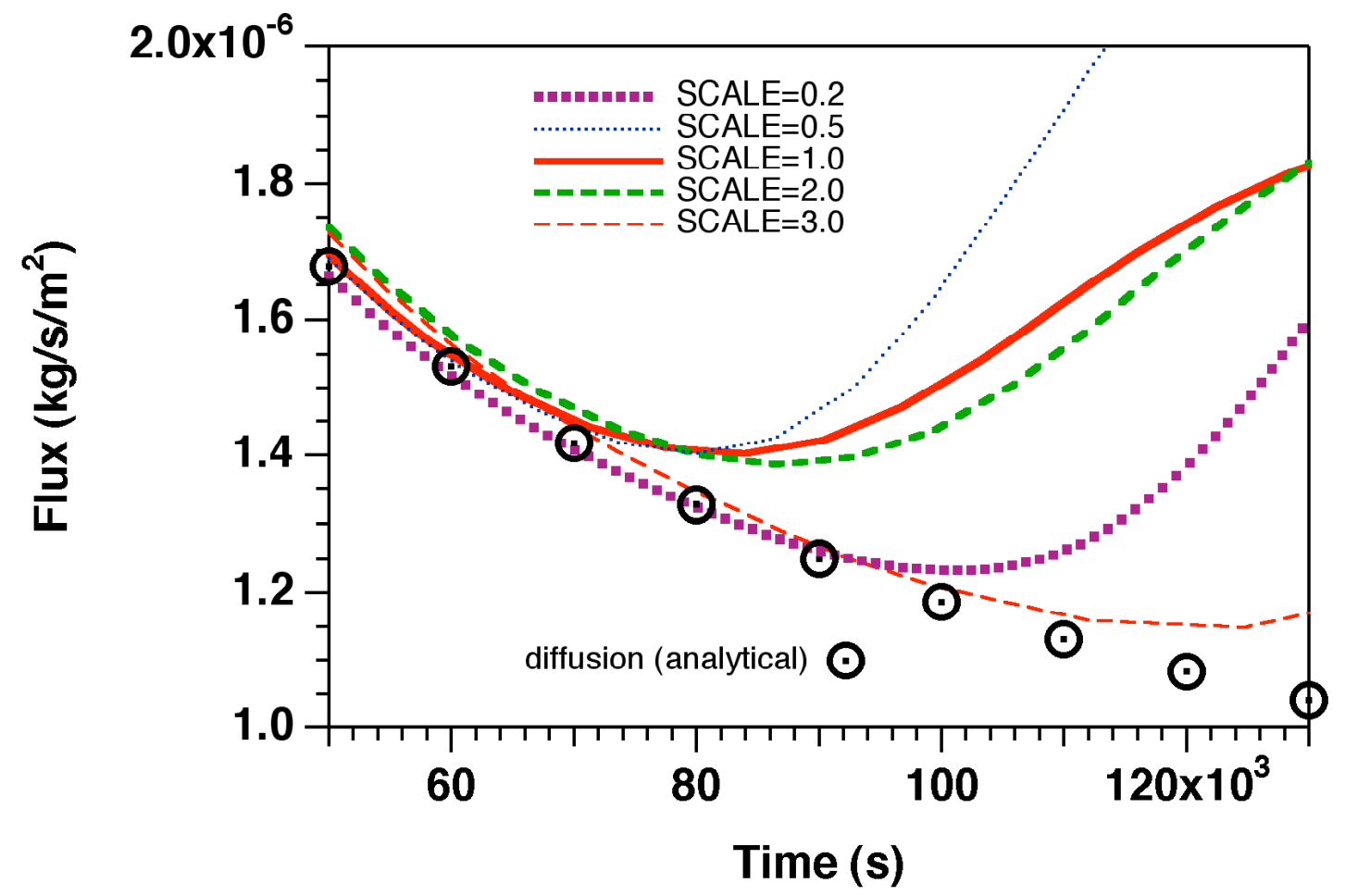

Figure 6. Simulated $\mathrm{CO} 2$ dissolution rates for a range of grid resolutions (see text). Dissolution rate from the analytical solution for diffusion only is also shown.

Simulated CO2 dissolution rates for $0.5 \leq \mathrm{SCALE} \leq 2.0$, corresponding to horizontal grid resolution of $5-20 \mathrm{~mm}$, begin to exceed diffusive flux at the same time of $t_{\mathrm{inc}}=7 \times 10^{4} \mathrm{~s}$. The good agreement for convective onset time between the simulations for $0.5 \leq$ SCALE $\leq 2.0$ indicates that there is little impediment for the development of the convective instability from horizontal grid resolution effects. In contrast, the coarser grid with SCALE $=3(30 \mathrm{~mm}$ resolution) is seen to suppress convection at early times. Somewhat surprisingly, onset of convection is also delayed for the finest grid resolution (SCALE $=0.2$, corresponding to a horizontal resolution of $2 \mathrm{~mm}$ ). We interpret this to mean that such fine horizontal gridding impedes development of fingers with the somewhat larger wave lengths that experience larger growth rates at early times. For the inferred onset time $t_{\text {inc }}=7 \times 10^{4} \mathrm{~s}$ and using the applicable problem parameters (Table 2), the coefficient in the theoretical expression Eq. (3) is calculated as $\mathrm{c}_{0}=1155.6$. 
Fig. 7 gives a more detailed view of the top of the flow domain for the case with SEED = $0.8, \operatorname{SCALE}=1$, and also shows the computational grid with vertical discretization refined to $\mathrm{h}=$ $1 \mathrm{~mm}$ near the top boundary. It is seen that the downward convection of CO2-ladden water is fed from four roots at the top of the flow domain, the rightmost of which splits into two as it is convecting downward. The horizontal yellow line indicates the position of the diffusive front at the incubation time, $\mathrm{L}_{\mathrm{inc}}=11.8 \mathrm{~mm}$, to which corresponds a dissolved $\mathrm{CO} 2$ mass fraction of $\mathrm{X}=$ 0.0236 at time $t_{\text {inc }}$. The interplay between diffusion and convection changes the distance from the top boundary at which a dissolved concentration of $X=0.0236$ occurs; at the time $t=101.6$ days for which Fig. 7 is plotted, this particular concentration corresponds to the top boundary of the dark blue region. Regions in which the diffusive boundary layer is compressed, due to upwelling water with low dissolved $\mathrm{CO} 2$, have increased concentration gradients, deliver larger diffusive

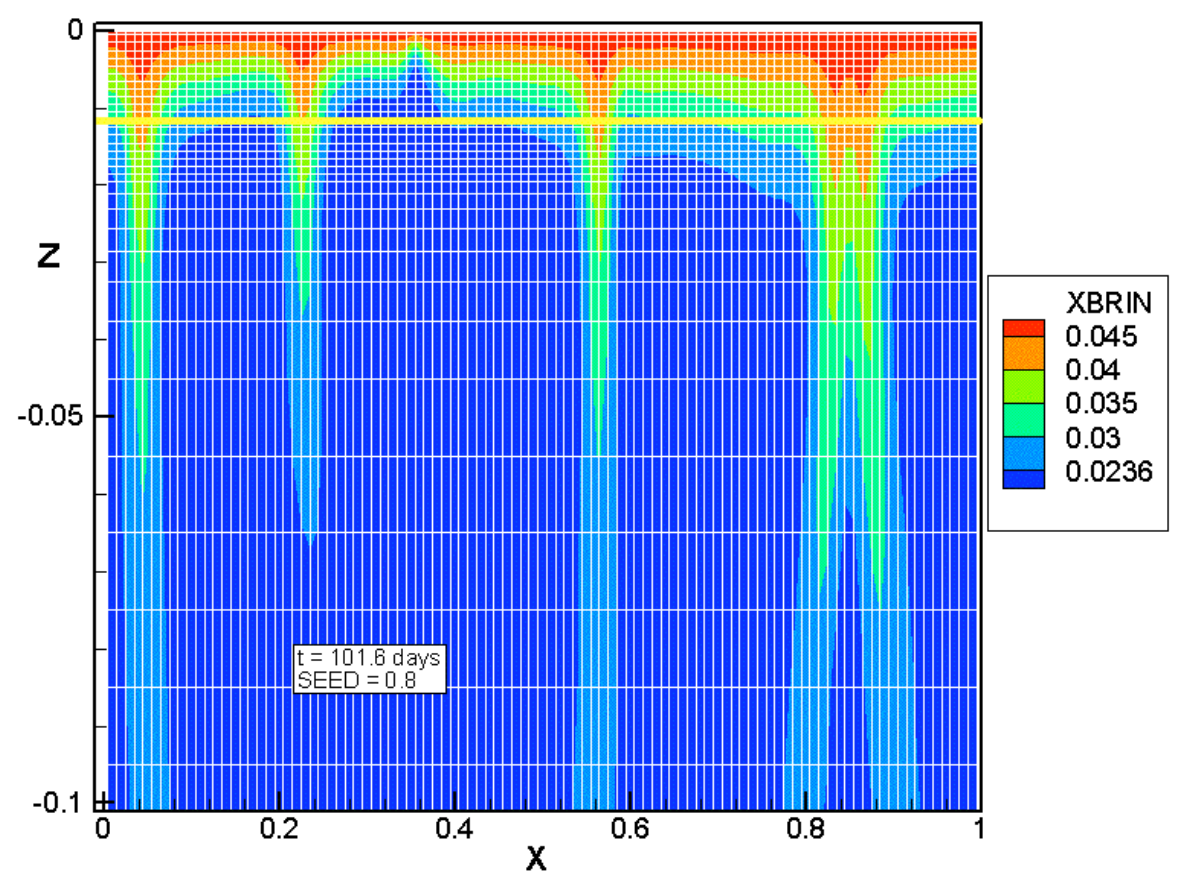

Figure 7. Dissolved CO2 mass fractions near the top of the flow domain for the Case 1 simulation with a random number $\operatorname{SEED}=0.8$ at time $\mathrm{t}=101.6$ days. Note the $10: 1$ vertical exaggeration. The horizontal yellow line corresponds to the diffusive boundary layer at incubation time, $|\mathrm{z}|=\mathrm{L}_{\mathrm{inc}}=\sqrt{\mathrm{Dt}_{\mathrm{inc}}}$, with a dissolved mass fraction of $\mathrm{X}=0.0236$. Gridding is also shown. All dimensions are in $\mathrm{m}$. 
flux locally, and thus function as the primary sources of dissolved CO2. An extreme example of one such region occurs near $\mathrm{x}=0.36 \mathrm{~m}$. Water convecting upwards in this region is diverted sideways near the top boundary; it then picks up additional solute from laterally offset regions with smaller $\mathrm{CO} 2$ concentration gradients, and eventually its density increases due to increased $\mathrm{CO} 2$ concentration to the point where it begins to convect downward. Subsequent simulations were carried out with the finer gridding of $\mathrm{h}=1 \mathrm{~mm}$ near the top and SCALE $=1$. The total number of grid blocks is 52,300 .

Fig. 8 gives space-averaged $\mathrm{CO} 2$ fluxes across horizons located at different distance from the top boundary, with data plotted on logarithmic (a) and linear time scales (b), respectively. This shows how $\mathrm{CO} 2$ transport progresses over time from the top boundary downward towards increasing distances. Fluxes in the top $2 \mathrm{~cm}$ quickly approach a quasi-steady behavior, and show only modest temporal variations of around $\pm 15 \%$. At deeper horizons fluxes continue to fluctuate more strongly, due to evolving convective activity.

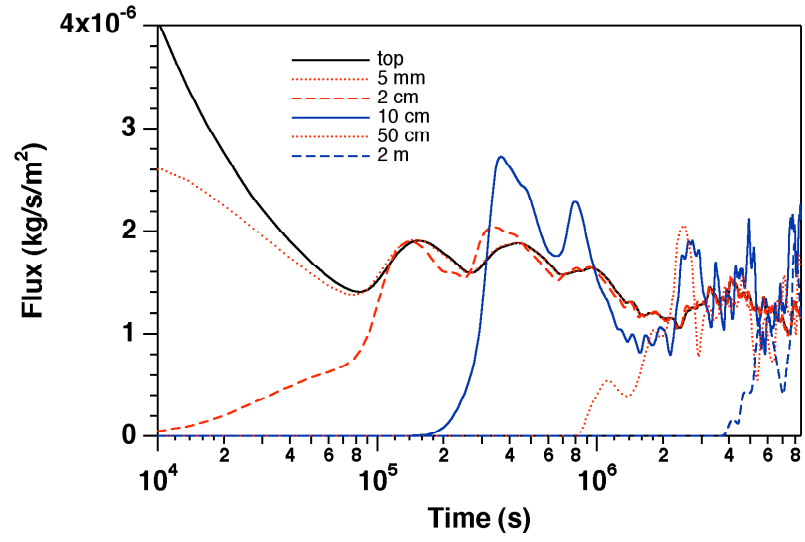

(a)

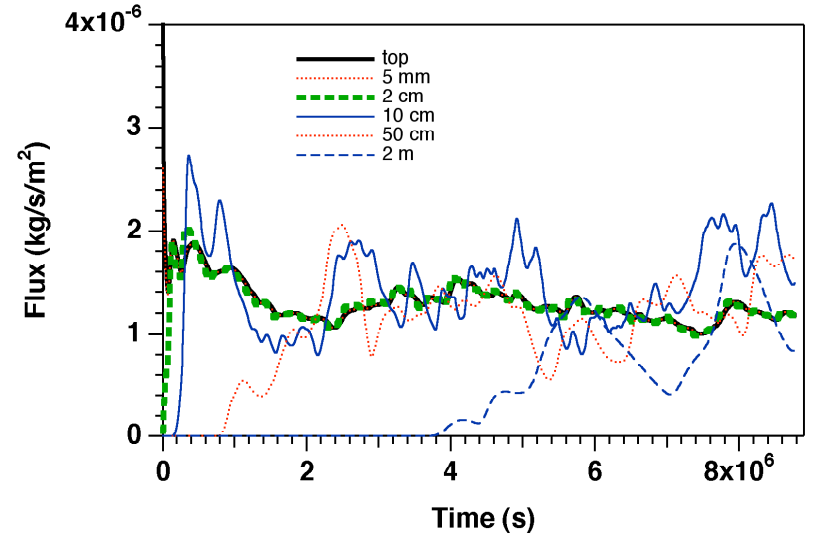

(b)

Figure 8. Total CO2 flux across horizons located at different distance from the top boundary for Case 1, showing data on logarithmic (a) and linear time scales (b), respectively. 


\subsection{Variations in Random Seeding}

Fig. 9 shows distributions of dissolved $\mathrm{CO} 2$ concentrations for three different random number seeds after 101.6 days of simulation time, just before significant $\mathrm{CO} 2$ concentrations reach the lower boundary. It is evident that convection patterns are quite different in detail, but share similar features overall.

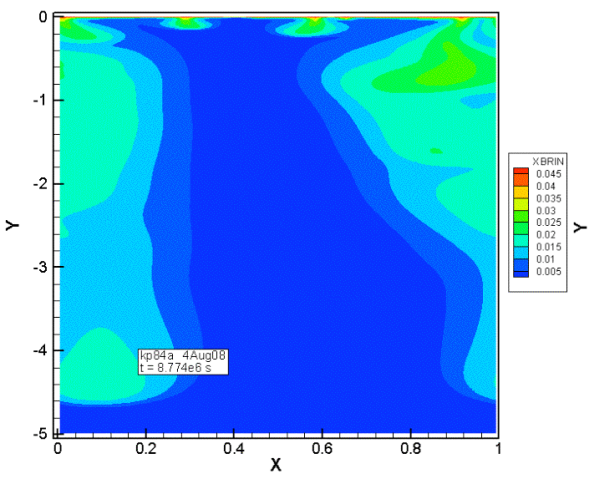

(a)

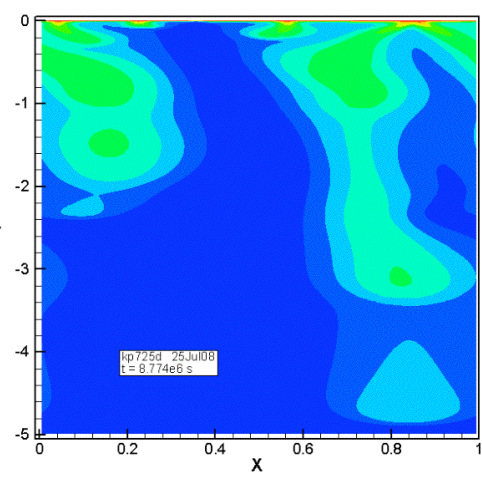

(b)

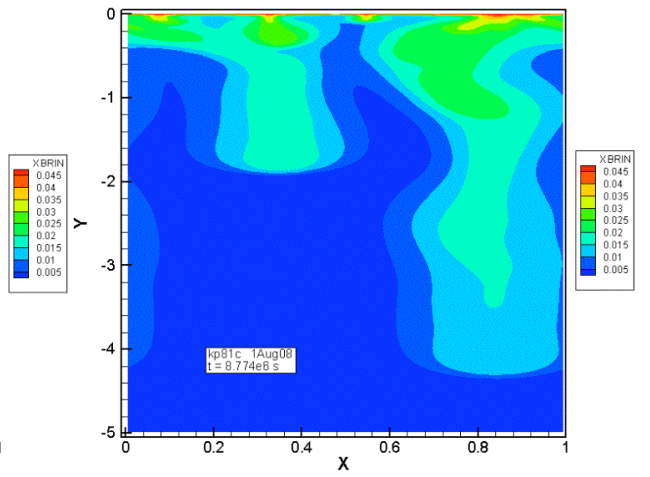

(c)

Figure 9. Simulated distribution of dissolved CO2 for Case 1 after 101.6 days for three different random number seeds to generate permeability heterogeneity $(a-0.7 ; b-0.8 ; c-0.9)$. Note the different horizontal and vertical scales.

The CO2 mass fluxes associated with the systems shown in Fig. 9 are plotted in Fig. 10 for the time period prior to significant $\mathrm{CO} 2$ concentrations evolving at the lower boundary. Convection begins at $\mathrm{t}_{\mathrm{inc}}=7 \times 10^{4} \mathrm{~s}$, at which time CO2 dissolution rate is $1.4 \times 10^{-6} \mathrm{~kg} / \mathrm{s} / \mathrm{m}^{2}$. Some rapid quasi-periodic variations occur at early-times as convective fingers grow and partially coalesce, followed by random variations of about $\pm 15 \%$ amplitude around an average flux of $1.3 \times 10^{-6} \mathrm{~kg} / \mathrm{s} / \mathrm{m}^{2}$. Apart from these variations, $\mathrm{CO} 2$ fluxes are seen to be essentially constant during the entire convection-dominated period, as long as no significant $\mathrm{CO} 2$ concentrations reach the lower boundary. This confirms earlier results by Hesse et al. (2006). We also note that differences between different random number realizations are of similar magnitude as temporal variations for a given realization. 

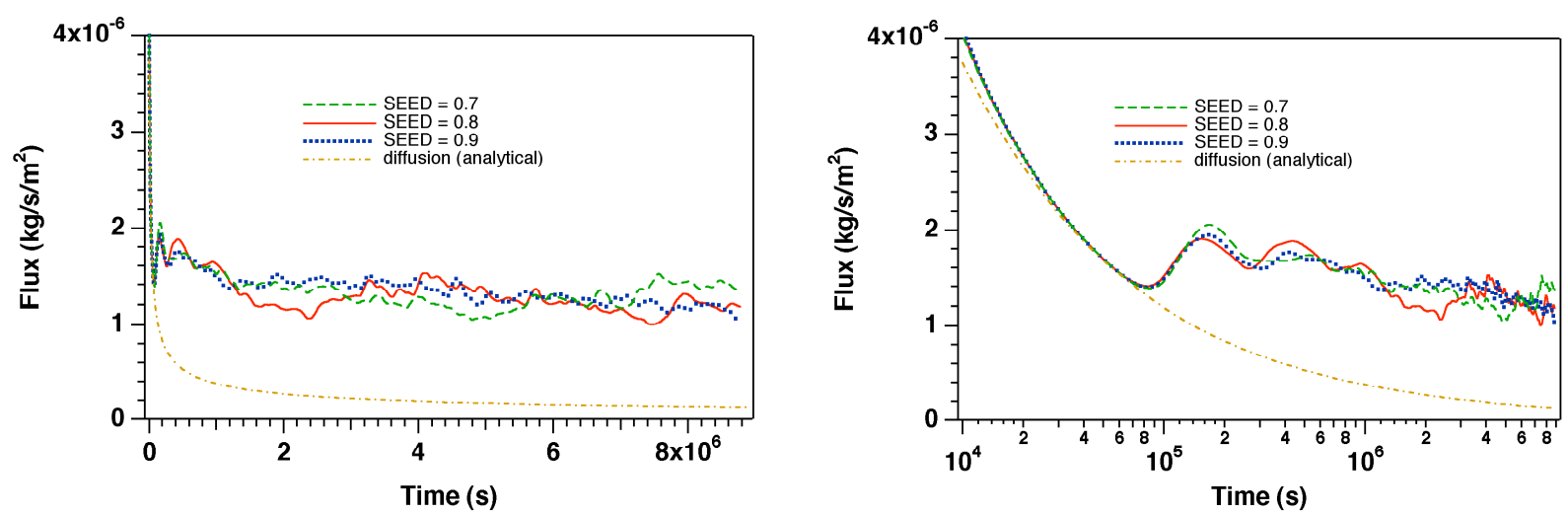

Figure 10. Simulated $\mathrm{CO} 2$ fluxes (dissolution rates) for Case 1 at the top boundary, for three different random number seeds, on linear (a) and logarithmic time scales (b).

Fig. 11 shows plots of the quantity F.M (Eq. 9), that remains constant for a purely diffusive process. This figure indicates that the onset time for convection is about $7 \times 10^{4} \mathrm{~s}$, and is practically indistinguishable for the different realizations with different random number seeds.

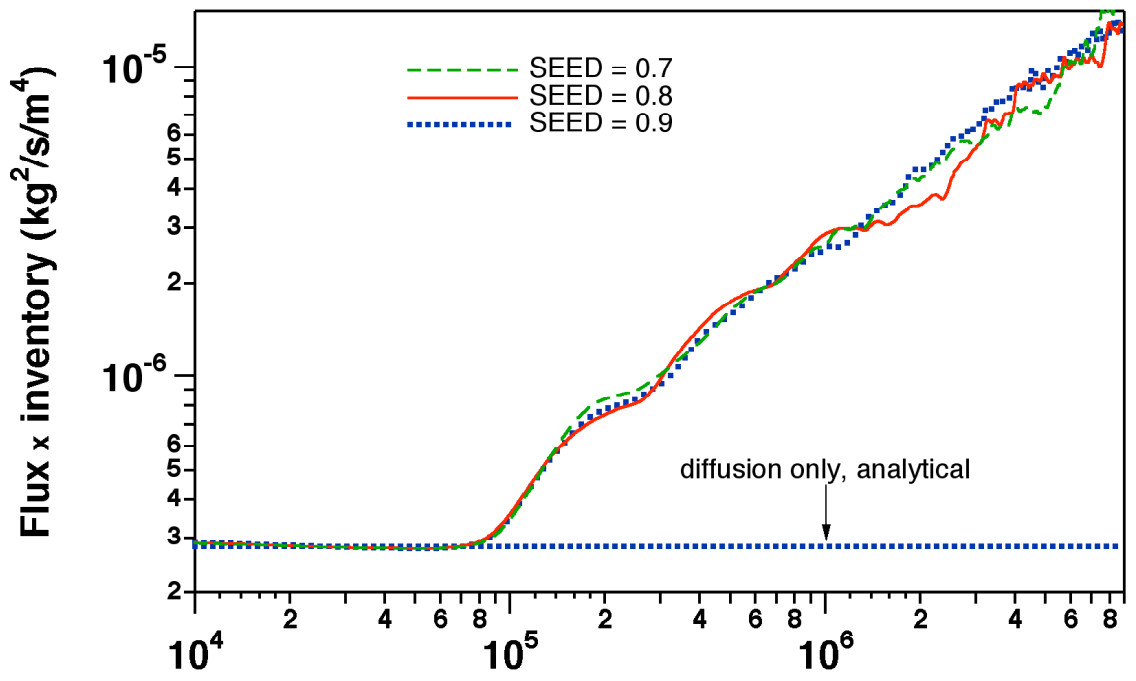

Time (s)

Figure 11. Parameter F.M (flux times mass inventory) for Case 1 simulations with different random number seeds. The dotted horizontal line indicates the behavior of a diffusive system without convection.

As demonstrated by the simulation results given above, the temporal evolution of $\mathrm{CO} 2$ dissolution rates proceeds through four distinct time periods, as follows. 
1) During the early period $\mathrm{t}<\mathrm{t}_{\mathrm{inc}}$, transport of $\mathrm{CO} 2$ away from the dissolution boundary occurs only by molecular diffusion, so that dissolution rate declines with time as $1 / \sqrt{ } \mathrm{t}$, while total dissolved inventory grows as $\sqrt{ } \mathrm{t}$.

2) This is followed by a period in which convective instabilities begin and grow, leading to a general increase in dissolution rates. These are modulated by significant quasi-periodic variations, as convective fingers partially coalesce and grow.

3) The third period is characterized by a continuous downward progression of convective fingers, while total dissolution rates remain constant except for modest random fluctuations of order $\pm 15 \%$.

4) The final period begins when dissolved $\mathrm{CO} 2$ reaches the lower boundary. At that time, dissolved $\mathrm{CO} 2$ concentrations begin to increase in the upwelling limbs of the convective process, leading to diminished buoyancy force and a gradual decline in dissolution rates.

The final equilibrium state of the system consists of constant dissolved $\mathrm{CO} 2$ concentrations throughout, equal to those applied at the top boundary.

\subsection{Variations in Boundary Conditions}

Here we present a series of simulations that were obtained for a random number seed of 0.8 , but employing different boundary conditions. The reference Case 1 discussed above has a no-flow boundary at the bottom of the domain $(\mathrm{z}=-5 \mathrm{~m})$. Variations of this case explored through numerical simulation experiments include (b) constant-pressure conditions at $\mathrm{z}=-5 \mathrm{~m}$, (c) use of an "unrolled" grid, in which the right hand side is not connected back to the left hand side, and (d) applying no flow boundary conditions at $\mathrm{z}=-4 \mathrm{~m}$. From the results given in Figs. 12-14, we make the following observations.

- details of convection patterns can be quite different when boundary conditions are changed;

- total dissolution rates at the top are $1.3 \times 10^{-6} \mathrm{~kg} / \mathrm{s} / \mathrm{m}^{2} \pm 15 \%$, and are not sensitive to boundary conditions and associated variations in convection patterns; average dissolution rates and their random scatter are similar to what was obtained for different random number seeds; 


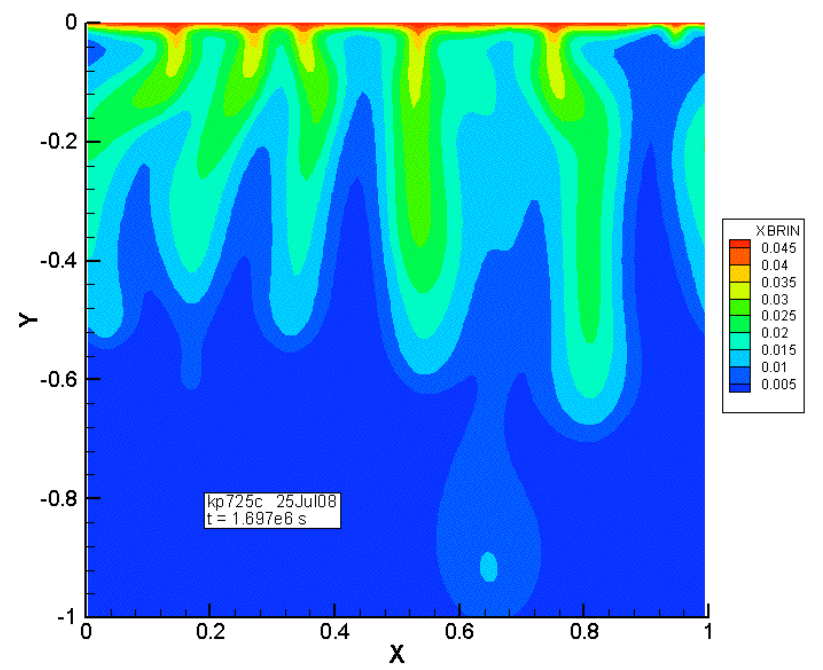

(a)

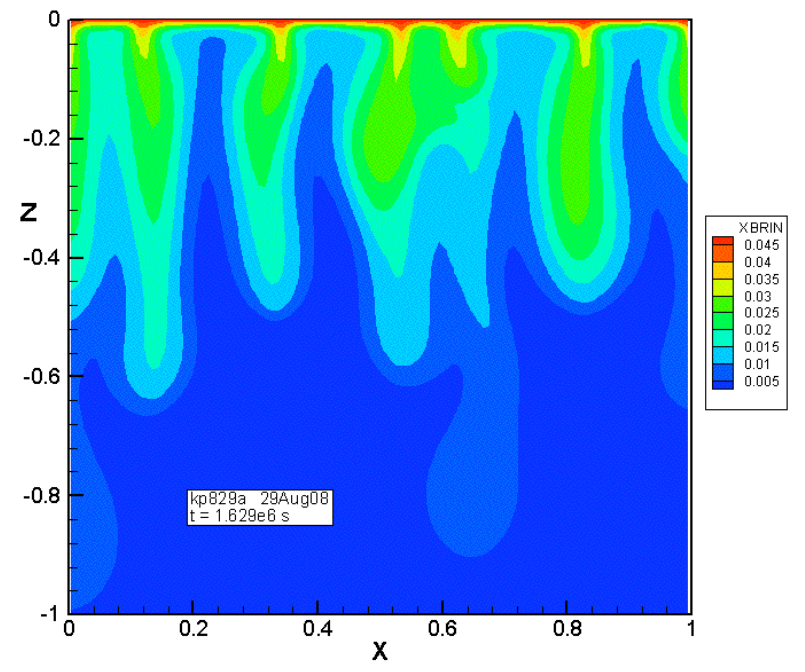

(c)

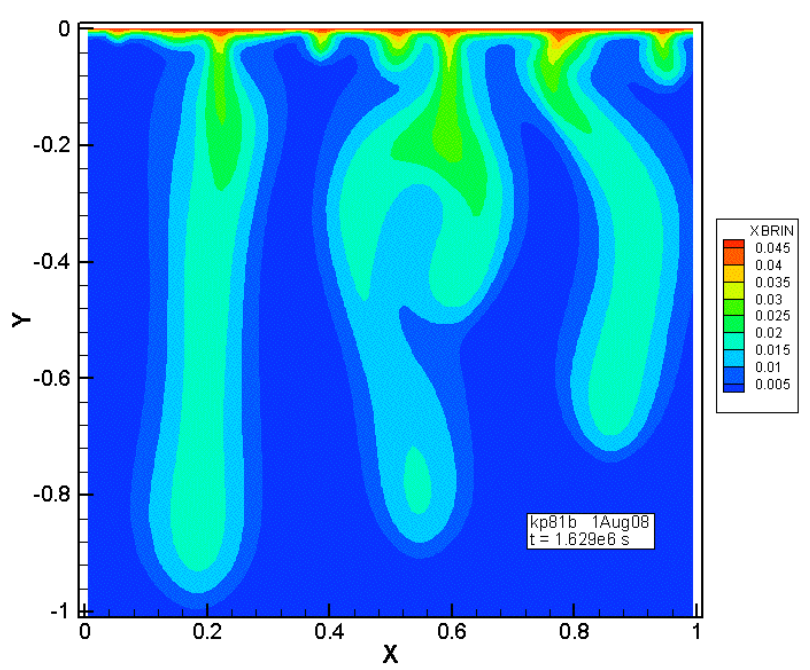

(b)

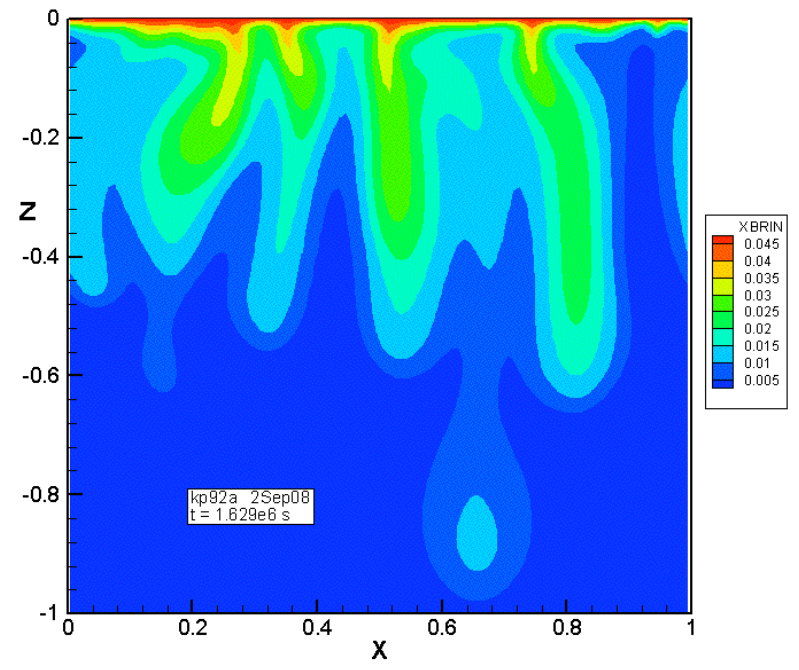

(d)

Figure 12. Simulated dissolved CO2 concentrations for Case 1 at time $t=1.629 \times 10^{6} \mathrm{~s}$ for (a) reference case, (b) constant $\mathrm{P}$ at $\mathrm{z}=-4.995 \mathrm{~m}$, (c) unrolled grid, and (d) no flow at $\mathrm{z}=-3.995 \mathrm{~m}$.

- there is considerable variability in the downward advancement of convective fingers, with a spread of about a factor 1.5 between fastest and slowest, akin to longitudinal dispersion;

- there can be considerable differences in the rate of advancement of the fastest finger;

- denser CO2-ladden fluid parcels can pinch off and become disconnected from convective fingers. 


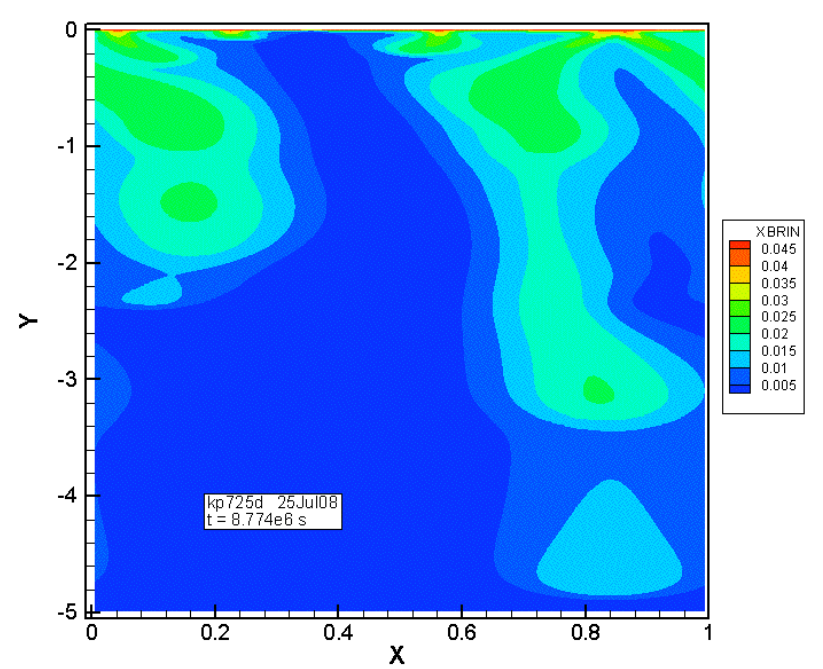

(a)

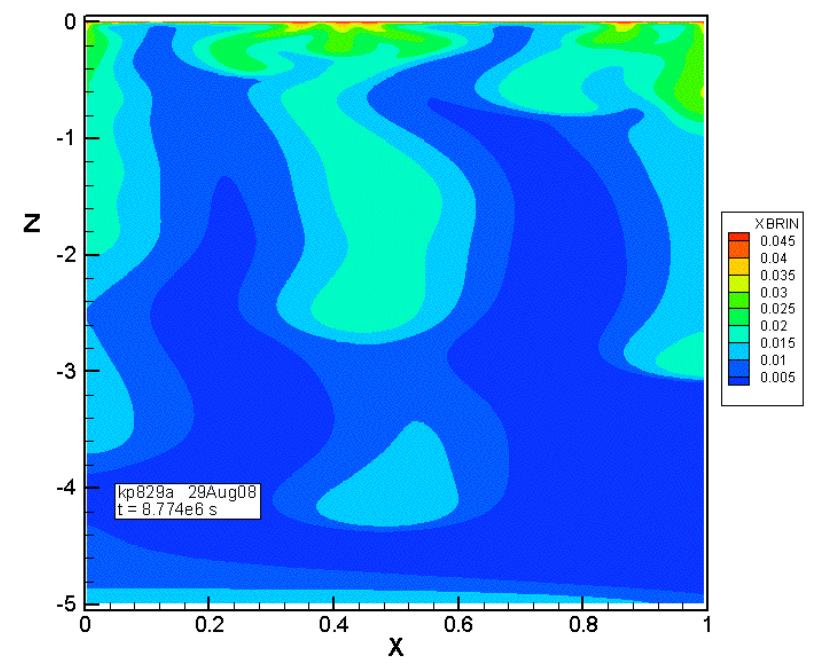

(c)

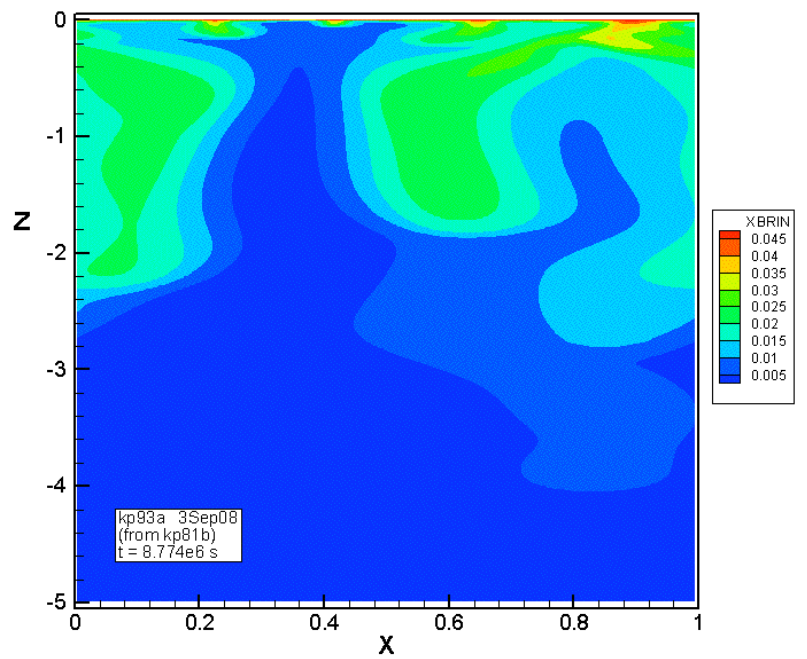

(b)

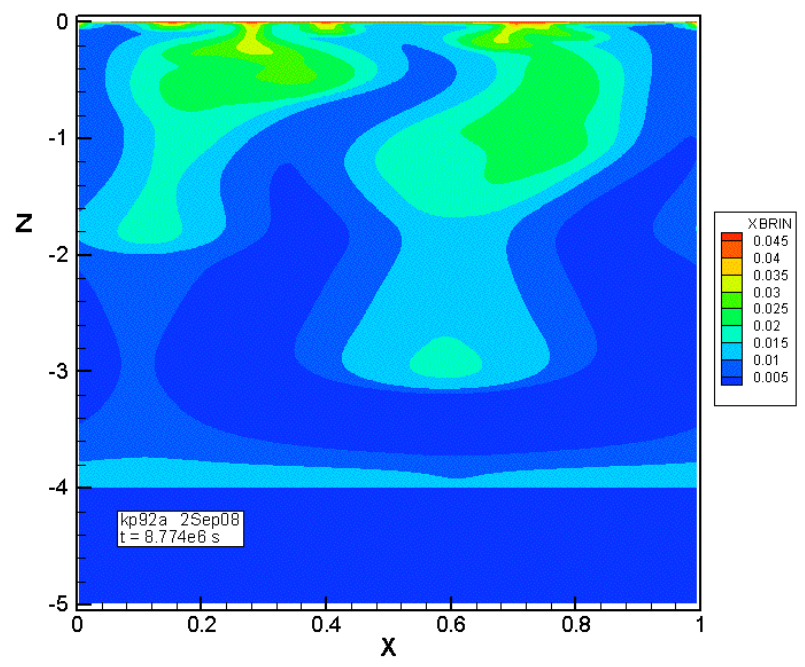

(d)

Figure 13. Simulated dissolved CO2 concentrations for Case 1 at time $t=8.774 \times 10^{6} \mathrm{~s}$ for (a) reference case, (b) constant $\mathrm{P}$ at $\mathrm{z}=-4.995 \mathrm{~m}$, (c) unrolled grid, and (d) no flow at $\mathrm{z}=-3.995 \mathrm{~m}$.

Overall, effects from variations in boundary conditions are similar to effects from different random number seeds. Such variations do not appear to induce any systematic changes in $\mathrm{CO} 2$ dissolution rates. 


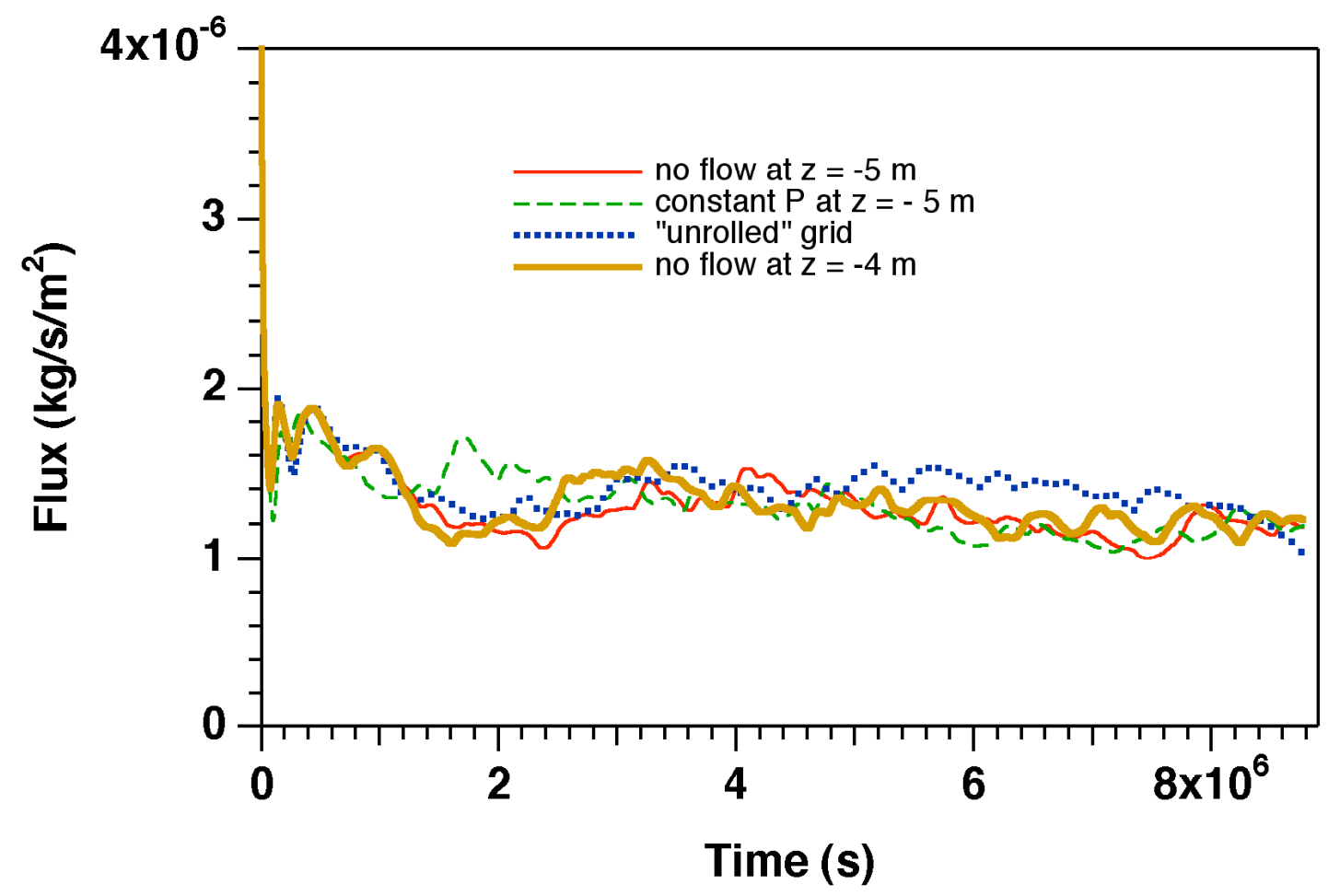

Figure 14. Simulated $\mathrm{CO} 2$ fluxes (dissolution rates) for Case 1 at the top boundary, for different conditions at the lateral and bottom boundaries.

\section{Results for Case 2 (low-k, large scale)}

Here we consider a permeability reduced by two orders of magnitude to $10^{-13} \mathrm{~m}^{2}$, which from Eqs. $(3,4)$ is expected to increase the incubation time by four orders of magnitude to $7 \times 10^{8}$ $\mathrm{s}$, and increase the thickness of the diffusive boundary layer at incubation time by two orders of magnitude to $1.18 \mathrm{~m}$. Additional smaller effects arise from changes in parameters D and $\phi$ (see Table 3), giving an estimated value for incubation time of $\mathrm{t}_{\mathrm{inc}}=1.56 \times 10^{8} \mathrm{~s}$, with a corresponding thickness of the diffusive boundary layer of $\mathrm{L}_{\mathrm{inc}}=0.39 \mathrm{~m}$.

This case was first run with a uniform grid spacing of $\mathrm{h}=0.1 \mathrm{~m}$ in both horizontal and vertical directions. Inspection showed very steep concentration gradients near the top, that appeared to be inadequately resolved by the $\mathrm{h}=0.1 \mathrm{~m}$ grid spacing. Accordingly, another run was made in which vertical grid spacing near the top was refined to $h=0.02 \mathrm{~m}$. Results are given in Figs. 15-17. 
Table 3. Parameter changes for Case 2 simulations.

\begin{tabular}{|l|l|}
\hline Fluid properties & \\
\hline diffusivity & $\mathrm{D}=2 \times 10^{-9}-->10^{-9} \mathrm{~m}^{2} / \mathrm{s}$ \\
\hline Formation properties & \\
\hline porosity & $\phi=0.3-->0.2$ \\
permeability & $\mathrm{k}=10^{-11}-->10^{-13} \mathrm{~m}^{2}$ \\
\hline Model domain & \\
\hline height & $\mathrm{H}=5-->10 \mathrm{~m}$ \\
width & $\mathrm{W}=1->20 \mathrm{~m}$ \\
\hline
\end{tabular}

Solute concentration patterns at time $t=32.2$ years are shown for the coarse and refined grids, respectively, in Fig. 15. In both cases there are five regions at the top of the domain that deliver CO2-ladden fluid downward, but other than that the concentration patterns are very different. Downward convection has advanced less in the coarser grid, as expected because coarser spatial discretization acts to suppress and delay convective activity. The concentration snapshots in Fig. 15 correspond to a time just before elevated $\mathrm{CO} 2$ concentrations reach the bottom boundary.

$\mathrm{CO} 2$ fluxes at the top of the domain are shown in Fig. 16, indicating incubation times of $2.3 \times 10^{8} \mathrm{~s}$ for the grid with uniform spacing of $\mathrm{h}=0.1 \mathrm{~m}$, and $1.9 \times 10^{8} \mathrm{~s}$ for the grid with refinement to $0.02 \mathrm{~m}$ at the top boundary. These numbers are comparable to but somewhat larger than the incubation time of $1.56 \times 10^{8} \mathrm{~s}$ estimated from Equation (3) using the Case-1 value of $\mathrm{c}_{0}$, suggesting that convective activity is somewhat suppressed and delayed due to spatial averaging from the limited grid resolution. In the finer grid, $\mathrm{CO} 2$ dissolution rate at onset time $\mathrm{t}_{\text {inc }}$ is $1.3 \times 10^{-8} \mathrm{~kg} / \mathrm{s} / \mathrm{m}^{2}$, two orders of magnitude smaller than in Case 1, in agreement with the scaling law Eq. (7). The coefficient in Eq. (3) for $\mathrm{t}_{\mathrm{inc}}=1.9 \times 10^{8} \mathrm{~s}_{\text {is }} \mathrm{c}_{0}=1411.5$. This is $22 \%$ larger than for the high-k Case 1, and about an order of magnitude larger than values suggested by EnnisKing and Paterson (2003b). The reason for these discrepancies are unknown.

It is remarkable that, in spite of the large differences in convection patterns, $\mathrm{CO} 2$ fluxes for the coarse and fine grid systems are quite similar, around $1.9 \times 10^{-8} \mathrm{~kg} / \mathrm{s} / \mathrm{m}^{2}$ during the main convective period (Fig. 16). Alternating periods of flux overshoot and undershoot between the 


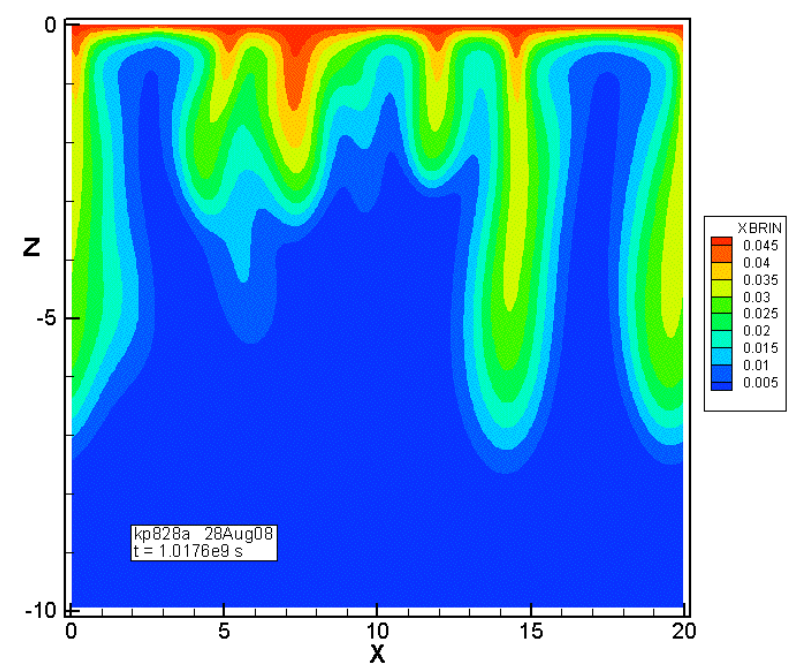

(a)

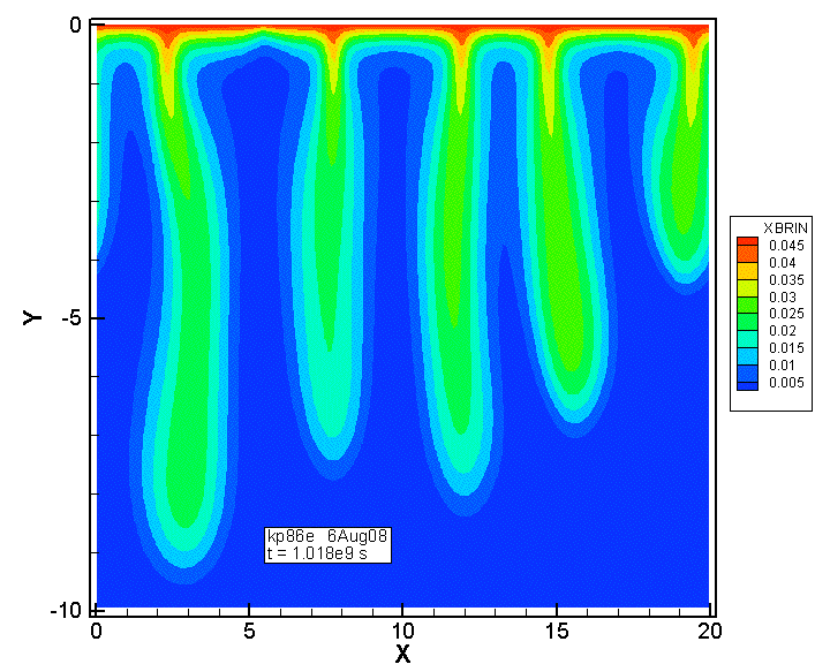

(b)

Figure 15. Simulated distributions of dissolved $\mathrm{CO} 2$ for Case 2 after 32.2 years for the coarse (left) and refined grid (right).

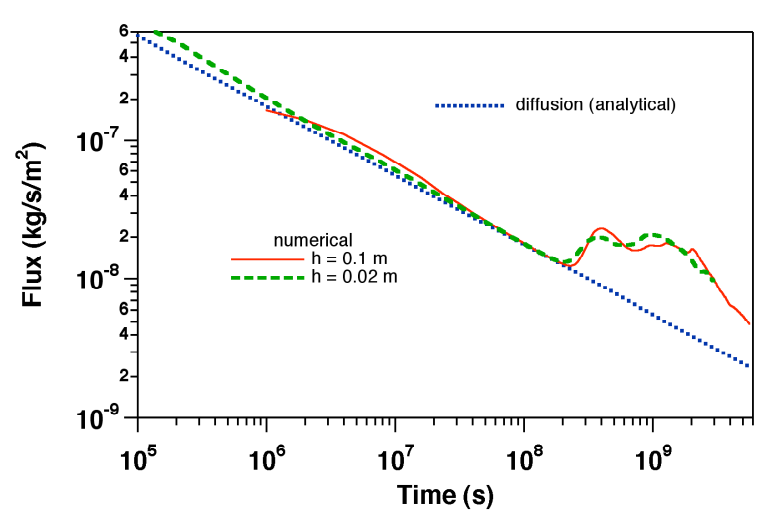

(a)

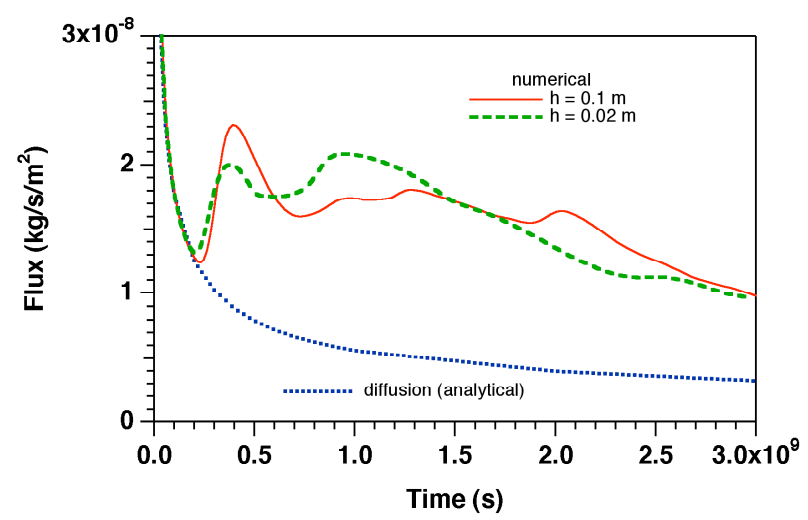

(b)

Figure 16. Simulated $\mathrm{CO} 2$ flux (dissolution rate) from the top boundary for the low-permeability Case 2 on logarithmic (a) and linear scales (b).

two grids tend to compensate each other, leading to very small differences in the evolution of total dissolved CO2 inventory (Fig. 17). To gain some perspective on the growth of dissolved inventory shown in Fig. 17, we note that the "end state" of the system reached for $\mathrm{t} \rightarrow \infty$ corresponds to a uniform dissolved $\mathrm{CO} 2$ concentration of $\mathrm{X}=\mathrm{X}_{0}=0.0493$, and a dissolved inventory per unit area of $\mathrm{H} \phi \rho \mathrm{X}_{0}=99.1 \mathrm{~kg} / \mathrm{m}^{2}$, about twice the inventory reached at $\mathrm{t}=3 \times 10^{9} \mathrm{~s}$. 


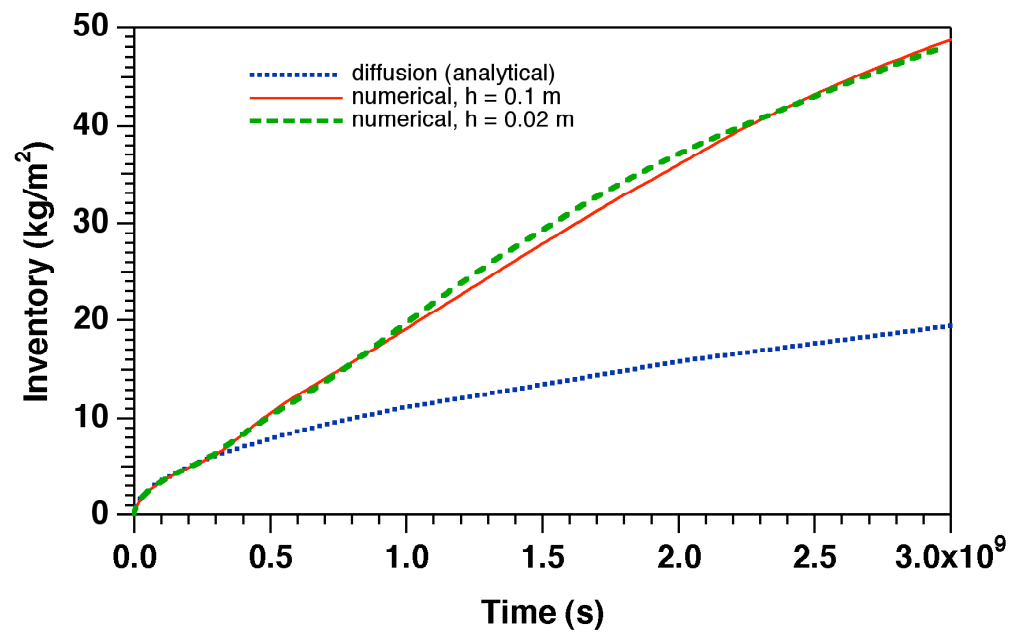

Figure 17. Simulated growth of dissolved $\mathrm{CO} 2$ inventory for Case 2, comparing results for different spatial resolution.

Fig. 18 shows $\mathrm{CO} 2$ dissolution rates for five different random number seeds to specify permeability heterogeneity. The different simulations agree very well with respect to incubation time. Significant differences occur in the subsequent period where convection is evolving, with some cases producing far greater excursions about the mean (approximately $1.9 \times 10^{-8} \mathrm{~kg} / \mathrm{s} / \mathrm{m}^{2}$ ) than others. Convective activity reaches the bottom boundary just after $10^{9} \mathrm{~s}$, and subsequently there is systematic decline, in some cases modulated by non-monotonic variations.

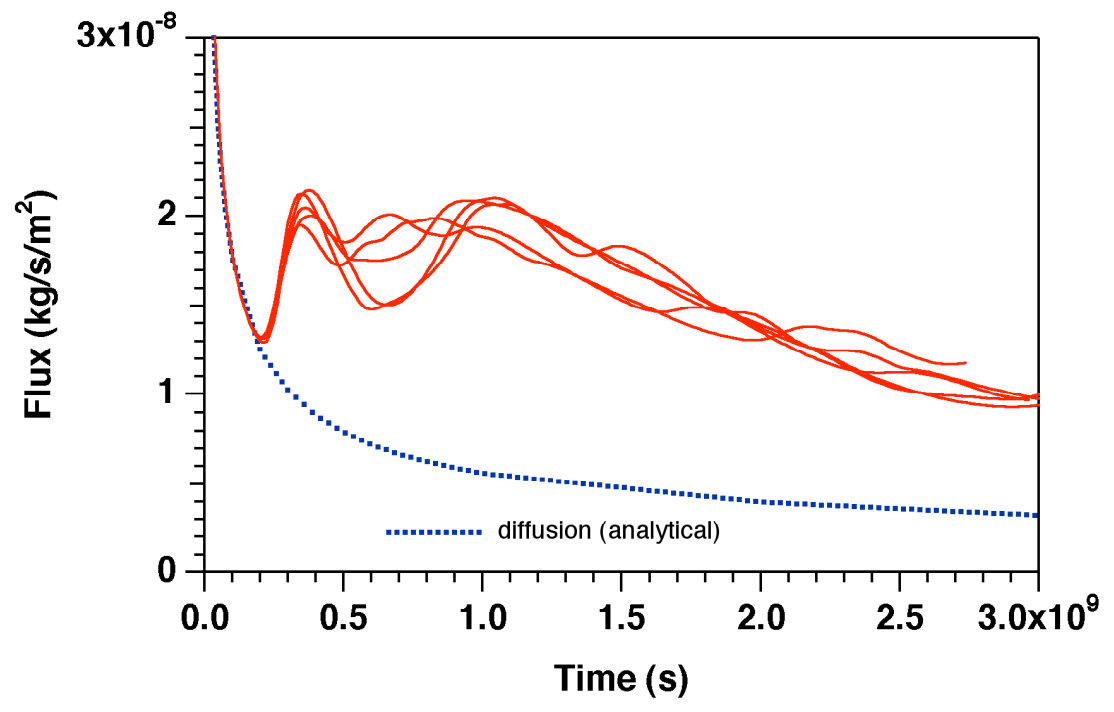

Figure 18. Simulated $\mathrm{CO} 2$ dissolution rates for Case 2 for 5 different choices of random seeding. 
An important difference in comparison to the high-permeability Case 1 is in the relative magnitude of incubation times, and times beyond which convection is affected by dissolved CO2 reaching the lower boundary of the flow system. Incubation times scale inversely proportional to permeability-squared (Eq. 3), while the time scale for convection to extend over a certain depth interval scales inversely proportional to permeability. The time required for convective fingers to reach the bottom of the flow domain is approximately $100 \mathrm{t}_{\text {inc }}$ in the high-k system, while in the low-k system it is only about $6 \mathrm{t}_{\mathrm{inc}}$. The time scale for evolution of convection is much slower for lower permeability, and the system is still "spinning up," with large excursions from the mean, when bottom boundary effects are beginning to be felt. This difference explains why in the lower-permeability case we do not have as much of an extended period with essentially constant dissolution rate (modulated by random variations) as had been seen in the high-permeability case.

\section{Discussion and Concluding Remarks}

Numerical modeling of dissolution-diffusion-convection (DDC) is challenging, due to the unstable nature of the governing processes, and the broad range of relevant space and time scales. The DDC process induces large concentration variations near the dissolution boundary. Resolving the accompanying concentration gradients by fine gridding is key to accurate numerical modeling. Assessing and controlling space discretization effects is a major challenge, as the most fundamental and commonly employed approach for this purpose - systematic grid refinement - is not workable when even small changes in gridding can completely alter global convection patterns. Instead we need to look for aggregate, integral measures of the DDC process, such as total $\mathrm{CO} 2$ dissolution rates, and examine their sensitivity to variations in problem parameters and numerical artifacts.

The convective activity induced by aqueous phase density increase from $\mathrm{CO} 2$ dissolution presents two complementary and even contradictory aspects. On the one hand, the pattern of convection is very "fickle" and changeable; not only in response to small modifications in problem specifications, such as different random seeding, or different boundary conditions, but also for "small" changes in the simulation process, such as altered numerical roundoff for different time stepping controls, and when different domain decompositions are applied in the 
linear equation solution. On the other hand, "integral" measures of the convective process, such as total solute flux across different horizons, are remarkably robust. Average solute flux across different horizons shows modest random fluctuations of typically $\pm 15 \%$ over time, and is not much affected by changes in boundary conditions.

The temporal evolution of $\mathrm{CO} 2$ dissolution rates proceeds through four distinct time periods, as follows.

1) During the early time period, transport of $\mathrm{CO} 2$ away from the dissolution boundary occurs by molecular diffusion only, with diffusivity of order $10^{-9} \mathrm{~m}^{2} / \mathrm{s}$ (Tewes and Boury, 2005), so that dissolution rate declines with time as $1 / \sqrt{ } \mathrm{t}$, while total dissolved inventory grows as $\sqrt{ }$.

2) This is followed by a period in which convective instabilities begin and grow, leading to a general increase in dissolution rates. These are modulated by non-monotonic variations, as convective fingers partially coalesce and grow.

3) The third period is characterized by a continuous downward progression of convective fingers, while total dissolution rates are constant with modest random fluctuations of order $\pm 15 \%$.

4) The final period begins when dissolved $\mathrm{CO} 2$ reaches the lower boundary. At that time, dissolved $\mathrm{CO} 2$ concentrations begin to increase in the upwelling limbs of the convective process, leading to diminished buoyancy force and a gradual decline in dissolution rates.

Our simulations confirm theoretically-derived scaling relations, according to which $\mathrm{CO} 2$ dissolution rate at onset time of convection is proportional to permeability, and independent of diffusivity and formation porosity.

\section{Acknowledgement}

The authors appreciate stimulating discussions with Jonathan Ennis-King, Tim Kneafsey, and Nicolas Spycher. We thank Hajime Yamamoto and Christine Doughty for a review of this report and the suggestion of improvements. This work was supported by the Office of Basic 
Energy Sciences under Contract No. DE-AC02-05CH11231 with the U.S. Department of Energy.

\section{References}

Audigane, P., I. Gaus, I. Czernichowski-Lauriol, K. Pruess and T. Xu. Two-Dimensional Reactive Transport Modeling of CO2 Injection in a Saline Aquifer at the Sleipner Site, North Sea, American Journal of Science, Vol. 307, pp. 974-1008, DOI 10.2475/07.2007.02, September 2007.

Carslaw, H. S., and J. C. Jaeger. Conduction of Heat in Solids, Oxford University Press, Oxford, England, Second Edition, 1959.

Ennis-King, J. and L. Paterson. Rate of Dissolution due to Convective Mixing in the Underground Storage of Carbon Dioxide, in J. Gale and Y. Kaya (Eds.), Greenhouse Gas Control Technologies, Volume 1, pp. 507-510, Elsevier, 2003 a.

Ennis-King, J. and L. Paterson. Role of Convective Mixing in the Long-Term Storage of Carbon Dioxide in Deep Saline Formations, paper SPE-84344, presented at Society of Petroleum Engineers Annual Fall Technical Conference and Exhibition, Denver, CO, October $2003 \mathrm{~b}$.

Ennis-King, J., I. Preston and L. Paterson. Onset of Convection in Anisotropic Porous Media subject to a rapid change in boundary conditions, Phys. of Fluids, Vol. 17, 084107, DOI: 10.1063/1.2033911, 2005.

Ennis-King, J., C. Green and K. Pruess. Effect of Vertical Heterogeneity on Long-Term Migration of $\mathrm{CO} 2$ in Saline Formations, presented at GHGT-9, Washington, DC, November 2008.

Garg, S.K. and D.R. Kassoy. Convective Heat and Mass Transfer in Hydrothermal Systems, in: L. Rybach and L.J.P. Muffler, (eds.), Geothermal Systems: Principles and Case Histories, John Wiley \& Sons, Chichester, New York, Brisbane, Toronto, 1981.

Hesse, M.A., H.A. Tchelepi and F.M. Orr, Jr. Natural Convection During Aquifer CO2 Storage, presented at GHGT-8, 8th International Conference on Greenhouse Gas Control Technologies, Trondheim, Norway, June 2006.

IPCC (Intergovernmental Panel on Climate Change), Special Report on Carbon Dioxide Capture and Storage, 2005.

Karypsis, G. and V. Kumar. METIS. A Software Package for Partitioning Unstructured Graphs, Partitioning Meshes, and Computing Fill-Reducing Orderings of Sparse Matrices, V4.0. Technical Report, Department of Computer Science, University of Minnesota, 1998.

Kimura, S., G. Schubert and J.M. Straus. Route to Chaos in Porous-Medium Thermal Convection, J. Fluid Mech., Vol. 166, pp. 305-324, 1986. 
Kneafsey, T.J. and K. Pruess. Laboratory Flow Experiments for Visualizing Convective Enhancement of CO2 Dissolution, submitted to Transport in Porous Media, 2008.

Lindeberg, E. and P. Bergmo. The Long-Term Fate of CO2 Injected into an Aquifer, in: J. Gale and Y. Kaya (eds.), Greenhouse Gas Control Technologies, pp. 489-494, Elsevier Science, Ltd., Amsterdam, The Netherlands, 2003.

Message Passing Forum, A Message-Passing Interface Standard, International Journal of Supercomputing Applications and High Performance Computing, 8(3-4), 1994.

Nordbotten, J.M. and M.A. Celia. Similarity Solutions for Fluid Injection into Confined Aquifers, J. Fluid Mech., Vol. 561, pp. 307-327, 2006.

Pruess, K. The TOUGH Codes-A Family of Simulation Tools for Multiphase Flow and Transport Processes in Permeable Media, Vadose Zone J., Vol. 3, pp. 738 - 746, 2004.

Pruess K. and N. Spycher. ECO2N - A Fluid Property Module for the TOUGH2 Code for Studies of $\mathrm{CO} 2$ Storage in Saline Aquifers, Energy Conversion and Management, doi:10.1016/j.enconman.2007.01.016, 2007.

Riaz, A., M. Hesse, H.A. Tchelepi and F.M. Orr Jr. Onset of Convection in a Gravitationally Unstable Diffusive Boundary Layer in Porous Media, J. Fluid Mech., Vol. 548, pp. 87$111,2006$.

Tewes, F. and F. Boury. Formation and Rheological Properties of the Supercritical CO2-Water Pure Interface, J. Phys. Chem. B, Vol. 109, No. 9, pp. 3990-3997, 2005.

Tuminaro, R.S., M. Heroux, S.A. Hutchinson, and J.N. Shadid. Official Aztec User's Guide, Ver 2.1, Massively Parallel Computing Research Laboratory, Sandia National Laboratories, Albuquerque, NM, 1999.

Weir, G.J., S.P. White and W.M. Kissling. Reservoir Storage and Containment of Greenhouse Gases, in: K. Pruess (ed.), Proceedings of the TOUGH Workshop '95, Lawrence Berkeley National Laboratory Report LBL-37200, pp. 233 - 238, Berkeley, CA, 1995.

Weir, G.J., S.P. White, and W.M. Kissling. Reservoir Storage and Containment of Greenhouse Gases, Transport in Porous Media, Vol. 23, pp. 37 - 60, 1996.

Wu, Y.S., K. Zhang, C. Ding, K. Pruess, E. Elmroth and G.S. Bodvarsson. An Efficient ParallelComputing Method for Modeling Nonisothermal Multiphase Flow and Multicomponent Transport in Porous and Fractured Media, Adv. Wat. Resour., Vol. 25, pp. 243 - 261, 2002.

$\mathrm{Xu}, \mathrm{X}$., S. Chen and D. Zhang. Convective Stability Analysis of the Long-term Storage of Carbon Dioxide in Deep Saline Aquifers, Adv. Wat. Resour., Vol. 29, pp. 397-407, 2006.

Yang, C. and Y. Gu. Accelerated Mass Transfer of CO2 in Reservoir Brine Due to DensityDriven Natural Convection at High Pressures and Elevated Temperatures, Ind. Eng. Chem. Res., Vol. 45, pp. 2430-2436, 2006. 
Zhang K., C. Doughty, Y.S. Wu and K. Pruess. Efficient Parallel Simulation of $\mathrm{CO}_{2}$ Geologic Sequestration in Saline Aquifers, Paper SPE 106026, Proceedings of the 2007 SPE Reservoir Simulation Symposium, Houston, Texas, February 2007.

Zhang, K., Y.S. Wu and K. Pruess. User's Guide for TOUGH2-MP - A Massively Parallel Version of the TOUGH2 Code, LBNL-315E, May 2008. 